\title{
An Extended Multiscale Finite Element Method (EMsFEM) Analysis of Periodic Truss Metamaterials (PTMM) Designed by Asymptotic Homogenization
}

\author{
Elias Jagielloa (iD, Pablo Andrés Muñoz-Rojas ${ }^{a^{*}}$ (iD \\ ${ }^{a}$ Departamento de Engenharia Mecânica, Centro de Ciências Tecnológicas, Universidade do Estado de Santa Catarina, Joinville, Brasil. \\ ejagiello@gmail.com, pablo.munoz@udesc.br \\ * Corresponding author
}

https://doi.org/10.1590/1679-78256240

\begin{abstract}
Asymptotic Homogenization ( $\mathrm{AH}$ ) and the Extended Multiscale Finite Element Method (EMsFEM) are both procedures that allow working on a structural macroscale that incorporates the effect of averaged microscopic heterogeneities, thus resulting in computationally efficient strategies. EMsFEM works directly on coupled finite micro and macroscales using numerically built discrete interpolation functions. Periodic Truss Metamaterials (PTMMs) are cellular materials formed by the periodic repetition of a truss-like unit cell and engineeringly tailored to show a given macroscopic response. In this work we analyze the numerical behavior of selected PTMMs that were designed for extreme Poisson ratios using AH theory. As a first issue, we study macroscopic structures made of finite unit cells and verify how close their average behavior coincides with the material properties predicted by $\mathrm{AH}$. For comparison, we solve the macroscopic plane stress associate problems that employ the elastic constitutive tensor obtained by $\mathrm{AH}$. The second issue is concerned with the ability of EMSFEM to reproduce the structural behavior of the full macro-micro model. We employ two versions of the EMsFEM, adopting linear ( $L B C$ ) and periodic (PBC) boundary conditions to build the numerical interpolation functions. The third and most important aspect discussed in this research concerns evaluation of the EMsFEM downscaled displacement fields. We observe that according to the layout of the AH designed unit cell, to the use of LBC or PBC and, depending on the boundary conditions present in the macroscopic problem, spurious downscaled displacements might occur. Such spurious displacements are due to excessive compliance of the corresponding unit cell and can be detected when building the numerical interpolation functions. We conclude that the layout optimization of PTMM using AH must be carefully interpreted and that EMsFEM is a good tool to detect a macroscopic excessively compliant response at an early design stage.
\end{abstract}

\section{Keywords}

Homogenization, Periodic Truss Materials, EMsFEM

\section{Graphical Abstract}
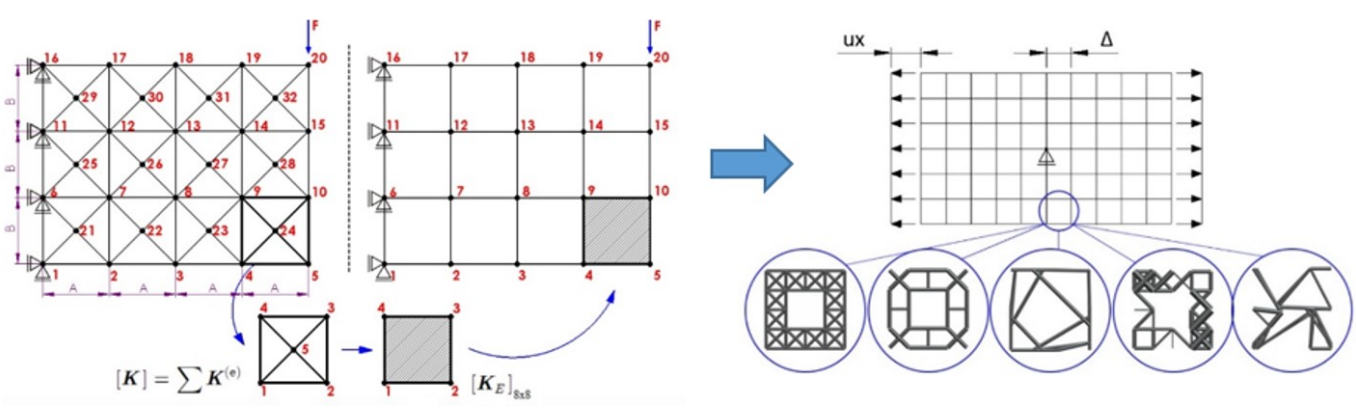

Received: August 25, 2020. In Revised Form: January 07, 2021. Accepted: January 07, 2021. Available online: January 29, 2021. https://doi.org/10.1590/1679-78256240

Latin American Journal of Solids and Structures. ISSN 1679-7825. Copyright (c) 2021. This is an Open Access article distributed under the terms of the Creative Commons Attribution License, which permits unrestricted use, distribution, and reproduction in any medium, provided the original work is properly cited. 


\section{INTRODUCTION}

In nature, it is frequent to find organic materials that evolved along time adapting to behave efficiently in multipurpose tasks. In the last decades, such materials have been studied with an engineering point of view, inspiring researchers to develop metamaterials for multifunctional technological applications (Ashby, 1983; Gibson and Ashby, 1997). These bio-inspired materials include the so-called periodic truss materials (PTMs), a special class of cellular materials made by the periodic repetition of unit cells composed of bar elements. As many porous materials, PTMs show a high stiffness/weight ratio and can be tailored for enhanced properties such as, impact energy absorption, bulk modulus, shear stiffness, negative Poisson ratio and many others. Lightness coupled to high capability to store strain energy are important features for automotive and aerospace industries, for instance (Yan et al., 2006). Moreover, owing to their spatial regularity, periodic cellular materials show little dispertion in their macroscopic properties, which can be an important engineering requirement. A good review on the development of engineeringly designed PTMs is given by Glaesener et al. (2019). Such structures have gained increased practical interest as additive manufacturing technologies have emerged (Plocher and Panesar, 2019). Figures 1 (a) and (b) display, respectively, an example of a 3D unit PTM cell and the corresponding porous material obtained by its periodic repetition.

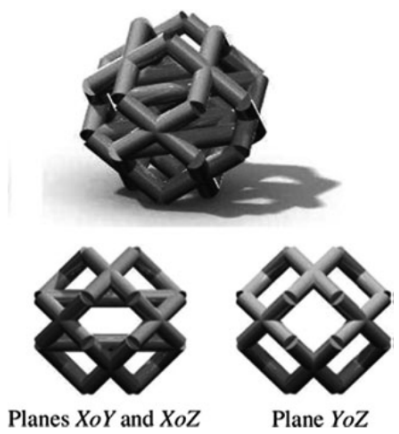

(a)

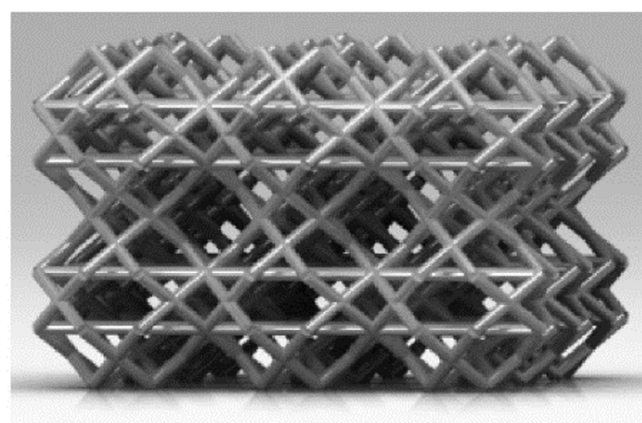

(b)

Figure 1 3D PTM. (a) unit cell (Muñoz-Rojas et al., 2010); (b) Corresponding material (Guth et al., 2012).

Engineering problems involving porous or spatially heterogeneous materials are usually solved using a discretization method such as the finite element method (FEM). However, the presence of two or more spacial scales may result in huge computational efforts. As an example, the analysis of a component made of the material shown in Fig. 1 (b) would require introduction of the whole set of bars directly into the numerical model, thus leading to very a large stiffness matrix (Liu and Lv, 2017). One way to handle this challenge is to use homogenization procedures, which replace the original unit cell by an averaged equivalent continuum material. Among the different approaches developed for homogenization along years, the most employed is certainly asymptotic homogenization (AH) although different alternatives exist, as discussed by Arabnejad and Pasini (2013).

The evolution of numerical tools directed to analyze multiscale microstructured materials has made possible also to design the microstructure of artificial materials aimed at optimum performance for multifunctional engineering tasks. The pioneering works in that sense go back to the 90's, when Sigmund $(1994,1995)$ merged truss sizing and continuum topology optimization with asymptotic homogenization to find optimum material distributions within the unit cell of periodic materials. Yan et al. (2006) optimized truss-made unit cells adopting joint positions as design variables. MuñozRojas et al. (2010) proposed the layout optimization (simultaneous cross sectional areas and coordinates as design variables) of truss-made unit cells for achieving optimum thermo-mechanical periodic truss metamaterials. Guth et al. $(2012,2015)$ optimized the layout of PTMMs including mechanical and thermal isotropic behavior as constraints in the optimization problem. All these works aimed at the optimization of material properties for a prescribed condition (shear stiffness, for instance) but they did not consider the pointwise response of a component made up of such material.

Concurrent topology optimization is a technique that allies the macroscopic topology optimization of a component with the optimization of its geometrical microstructure (second scale). Hence, it deals directly with the multiscale nature of the engineering problem. In its usual formulation, for manufacturability reasons, the microstructure is optimized to be constant in the whole component domain (Zhang and Sun, 2006). Liu et al. (2008) proposed a more comprehensive approach in which the component domain can be subdivided into design elements with independent material microstructures. Two limiting cases arise: if there is only one design element for the whole domain, we recover the usual macroscopic topology optimization; on the other hand, if we employ an infinite number of design elements, the approach 
particularizes to the usual unit cell material design optimization procedure. Adopting a finite number of design elements leads to different results where the scale effect between macro and microscale becomes clear. Cheng et al. (2017) and Wang at al. (2020) address the problem of concurrent optimization with variable relative density cells, aiming at manufacturability with additive manufacturing technologies. Li et al. (2020) presented a conformal gradient lattice optimization in which the relative density and orientation of the optimized cell changes according to its location within the domain.

In the brief review afore described, all the works employ asymptotic homogenization. As an alternative to analyze efficiently materials that show heterogeneities at a microscale, Zhang et al. (2010 a, b) proposed the Extended Multiscale Finite Element Method (EMsFEM), in which the finite nature of the microscale is directly taken into consideration, so both traditional (Yan et al., 2015) and concurrent optimization (Jun et al. 2015) can be handled in a natural way.

In this work, we use the EMsFEM to study structures made up of PTMMs that were optimized for maximum and minimum Poisson ratios using asymptotic homogenization (Guth et al., 2012). We verify that as asymptotic homogenization assumes an infinitesimal microscale (unit cell dimension) and is strain-driven, it is uncapable to detect important phenomena that can be naturally captured with EMsFEM. In particular, we focus in the problems caused by excessive compliance shown by some PTMMs, which can be detected in the EMsFEM by the presence of unexpected large downscaled displacements within unit cells when the material is subjected to external loading. While this phenomenon is not likely to occur in continuum topology optimization, care must be taken when the unit cell optimization is performed using pin-jointed truss elements.

We organize this article in the following way: Sections 2 and 3 briefly review the theoretical bases of AH and EMsFEM for PTMs; Section 4 presents the unit cells previously obtained by Guth et al. (2012) for extreme Poisson ratios; in Section 5 we show the EMsFEM numerical interpolation functions obtained for each of the unit cells presented in Section 4; in Section 6 we discuss numerical results comparing the AH and EMsFEM procedures. Finally, in Section 7 we close the article with concluding remarks.

\section{ASYMPTOTIC HOMOGENIZATION (AH)}

In this method, the macro and microscales ( $x$ and $y$ respectively) are related by $y=x / \varepsilon$ where the parameter $\varepsilon$ tends to zero, so that the microscale is assumed to be infinitesimal with respect to the macroscale. Furthermore, at a given macroscopic point $P$, the microscale is idealized by a Representative Volume Element (RVE) or unit cell. Figure 2 illustrates a situation where the unit cell is composed of 8 bars, connecting 5 points. Each bar has its own Young modulus and cross section area, and the rest of the cell domain is void, so at this scale the domain is clearly heterogeneous. Asymptotic homogenization allows to model the average material behavior of the cell (represented in Fig. 2 by the homogenized elastic tensor $\boldsymbol{E}^{H}$ ), and replace it at the macroscopic point $P$.

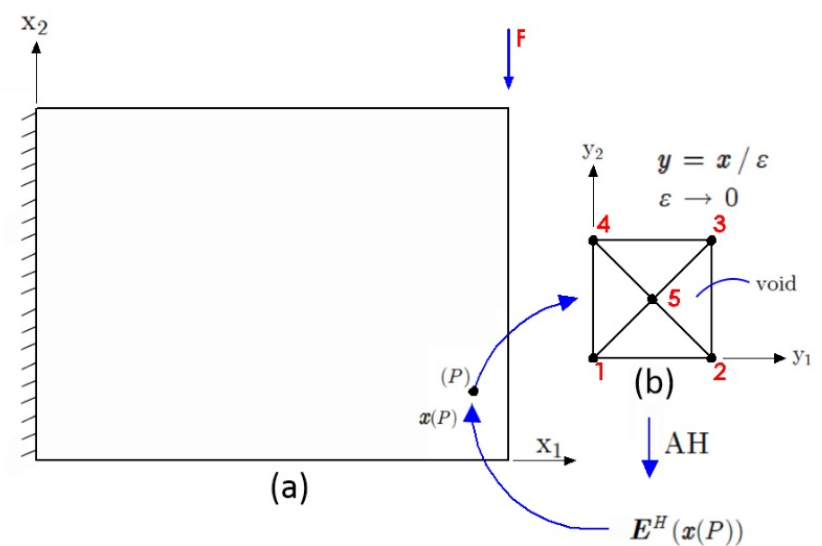

Figure 2 A continuum domain with microscopic heterogeneities modeled by asymptotic homogeneization. The unit cell is composed of a truss-like structure.

In order to obtain the homogenized properties, we expand the displacement field in the two scales $x$ and $y$ as

$u(x, y)=u_{0}(x)+\varepsilon u_{1}(x, y)$ 
where $u_{1}$ is periodic in the dimension $Y$ of the unit cell, and índices 0 and 1 refer to macro and micro contributions. We assume no traction on the internal borders of the unit cell, so that the principle of virtual work is expressed as

$$
\int_{\Omega} \boldsymbol{\sigma}: \boldsymbol{\delta} \varepsilon d \Omega-\int_{\Omega} \boldsymbol{b} \cdot \boldsymbol{\delta} \boldsymbol{u} d \Omega-\int_{\Gamma} \boldsymbol{t} \cdot \boldsymbol{\delta} \boldsymbol{u} d \Gamma=0 \quad \forall \boldsymbol{\delta} \boldsymbol{u} \in V_{\Omega}
$$

where $\sigma$ and $\varepsilon$ are the stress and strain tensors, respectively, $b$ is the body force, $t$ is the surface force acting on $\Gamma$ (the border of $\Omega$ ), $\delta \boldsymbol{u}$ is the virtual displacement field and $V_{\Omega}$ is the set of kinematically admissible set of virtual displacements.

Now we define the operator

$\partial_{x}=\frac{1}{2}\left(\frac{\partial(\cdot)_{k}}{\partial x_{l}}+\frac{\partial(\cdot)_{l}}{\partial x_{k}}\right)$

and after some algebraic manipulations we obtain the macroscopic equation (Muñoz-Rojas et al., 2010)

$$
\int_{\Omega} \partial_{x} \boldsymbol{\delta} \boldsymbol{u}_{0}: \boldsymbol{E}^{H}: \boldsymbol{\partial}_{x} \boldsymbol{u}_{0} d \Omega-\int_{\Omega} \boldsymbol{b} \cdot \boldsymbol{\delta} \boldsymbol{u}_{0} d \Omega-\int_{\Omega} \boldsymbol{t} \cdot \boldsymbol{\delta} \boldsymbol{u}_{0} d \Gamma=0
$$

where

$$
\boldsymbol{E}^{H}=\frac{1}{|\boldsymbol{Y}|} \int_{Y} \boldsymbol{E}:\left(\boldsymbol{I}-\boldsymbol{\partial}_{\boldsymbol{y}} \boldsymbol{\chi}\right) d Y
$$

is the homogeneized (or macroscopic) elastic tensor. Equation (5) can also be presented, in component form, as

$$
E_{i j k l}^{H}(\boldsymbol{x})=\frac{1}{|\boldsymbol{Y}|} \int_{Y}\left(E_{i j k l}-E_{i j p q} \frac{\partial \chi_{p}^{k l}}{\partial \chi_{q}}\right) d Y
$$

where $\chi_{p}^{k l}$ is the set of characteristic displacements, obtained as the solution of the microscopic equation

$$
\int_{Y} \partial_{y} \boldsymbol{\delta} \boldsymbol{u}_{1}: \boldsymbol{E}: \boldsymbol{\partial}_{y} \chi d Y=\int_{Y} \boldsymbol{\partial}_{y} \boldsymbol{\delta} \boldsymbol{u}_{1}: \boldsymbol{E}: \boldsymbol{I} d Y=0
$$

More details can be found in Guedes and Kikuchi (1990), Hassani and Hinton (1998) and Muñoz-Rojas et al. (2010).

\subsection{Numerical determination of $\chi$}

For PTMs, using Voigt compact notation, a numerical approximation of $\chi$ can be obtained using linear 3D bar finite elements to solve Eq. (7) (Yan et al., 2006)

$$
\left[\boldsymbol{K}^{A H}\right] \underbrace{\left[\left\{\chi^{11}\right\}\left\{\boldsymbol{\chi}^{22}\right\}\left\{\boldsymbol{\chi}^{12}\right\}\right]}_{[\chi]}=\underbrace{\left[\left\{\boldsymbol{P}^{11}\right\}\left\{\boldsymbol{P}^{22}\right\}\left\{\boldsymbol{P}^{12}\right\}\right]}_{[\boldsymbol{P}]}
$$

or

$$
\left[\boldsymbol{K}^{A H}\right][\chi]=[\boldsymbol{P}]
$$

with 


$$
\left[\boldsymbol{K}^{A H}\right]=\sum_{e} \int_{\Omega^{e}}[\boldsymbol{B}]^{T}[\boldsymbol{D}][\boldsymbol{B}] d Y^{e} \text { and }[\boldsymbol{P}]=\sum_{e} \int_{\Omega^{e}}[\boldsymbol{B}]^{T}[\boldsymbol{D}][\boldsymbol{I}] d Y^{e}
$$

where $[\boldsymbol{B}]$ is the strain-displacement matrix, $[\boldsymbol{D}]$ is the local 1-D constitutive matrix of the bar element rotated to the global system of reference, $\left[\boldsymbol{K}^{A H}\right]$ is the homogenized global stiffness matrix and $[\boldsymbol{P}]$ is a matrix containing, in each column, one of the global load cases that arise in the homogenization process. Each global load case corresponds to a unit strain applied in a given direction on the unit cell (Yan et al, 2006; Muñoz-Rojas et al., 2010).

\subsubsection{Periodic boundary conditions for $\mathrm{AH}$}

In order to solve Eq. (8) we apply periodic boundary conditions at the borders of the unit cell, so that displacements at opposite sides are constrained to be equal. In this work we adopt the condensation method, which demands classifying all the degrees of freedom (components of vector $\{\chi\}^{k l}$ in Eq. (8)) as being either independent (domain and boundary master degrees of freedom) or dependent (boundary slave degrees of freedom).

Consider the example in Fig. 3. The unit cell displayed has p nodes on both upper and lower faces, and $q$ nodes on each of the lateral sides.

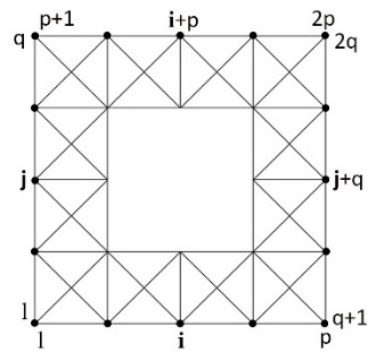

Figure 3 Periodic boundary conditions used for $A H$.

Dropping the upper indices $k l$ in $\{\chi\}^{k l}$, the kinematic constraints that relate the displacements on the upper and lower sides of the unit cell are given by

$$
\{\boldsymbol{\chi}\}_{i+p}=\left\{\begin{array}{l}
\boldsymbol{\chi}_{u} \\
\boldsymbol{\chi}_{v}
\end{array}\right\}_{i+p}=\{\boldsymbol{\chi}\}_{i}=\left\{\begin{array}{c}
\boldsymbol{\chi}_{u} \\
\boldsymbol{\chi}_{v}
\end{array}\right\}_{i} \forall i \in \Gamma_{\text {bottom_nodes }_{-}}=\{1,2, \ldots, p\}
$$

and the constraints that relate displacements on the right and left sides, by

$$
\{\boldsymbol{\chi}\}_{j+q}=\left\{\begin{array}{l}
\boldsymbol{\chi}_{u} \\
\boldsymbol{\chi}_{v}
\end{array}\right\}_{j+q}=\{\boldsymbol{\chi}\}_{j}=\left\{\begin{array}{l}
\boldsymbol{\chi}_{u} \\
\boldsymbol{\chi}_{v}
\end{array}\right\}_{j} \forall j \in \Gamma_{\text {left_nodes }}=\{1,2, \ldots, q\}
$$

where $\chi_{u}$ and $\chi_{v}$ stand for displacements in the $\boldsymbol{x}$ and $\boldsymbol{y}$ directions, for each of the columns of $[\boldsymbol{\chi}]$. The degrees of freedom of the nodes in the sets $\Gamma_{\text {left_nodes }}$ and $\Gamma_{\text {bottom_nodes }}$ are named independent or master degrees of freedom. The degrees of freedom of the remaining nodes on the boundary are called dependent or slaves since, as shown in Eqs. (12) and (13), they are linked to the master ones by kinematic constraints that can be easily set using a Boolean matrix $\left[T^{\Gamma}\right]$, such that

$\{\boldsymbol{\chi}\}_{\Gamma}=\left[\boldsymbol{T}^{\Gamma}\right]\{\boldsymbol{\chi}\}_{\Gamma m}$, where

$\{\boldsymbol{\chi}\}_{\Gamma}=\left\{\begin{array}{l}\{\boldsymbol{\chi}\}_{\Gamma_{m}} \\ \{\boldsymbol{\chi}\}_{\Gamma s}\end{array}\right\}=\left\{\begin{array}{l}\chi_{\Gamma_{\Gamma_{\text {Left_Nodes }}}} \\ \chi_{\Gamma_{\Gamma_{\text {Bottom_Nodes }}}} \\ \chi_{\Gamma_{\Gamma_{\text {dependent_Nodes }}}}\end{array}\right\}$. 
Considering Eq. (14), in the particular case where there are $n$ boundary degrees of freedom and 1 constraint equation (one degree of freedom), the Boolean matrix $\left[\boldsymbol{T}^{\Gamma}\right]$ will have dimension $n \times \mathbf{x}(n-1)$. In the example given in Fig. 4 , the constraint imposes the value of the degree of freedom $J$ to be equal to the value of the degree of freedom $I$. Generalization of this example to $n c$ constrained degrees of freedom yields

$$
\left[\boldsymbol{T}^{\Gamma}\right]=\left[\begin{array}{c}
{[\boldsymbol{I}]_{(n-n c) \mathrm{x}(n-n c)}} \\
{\left[\boldsymbol{T}_{d}\right]_{(n c) \mathrm{x}(n-n c)}}
\end{array}\right],
$$

where ordering has been reorganized by gathering together independent and dependent degrees of freedom.

$$
\left[\boldsymbol{T}^{\Gamma}\right]=\left[\begin{array}{ccccccccccc}
1 & 2 & \cdots & I & \cdots & J & \cdots & n-1 & \\
- & - & - & - & - & - & - & - & & \\
1 & 0 & \vdots & 0 & \vdots & 0 & \vdots & 0 & \mid & 1 \\
0 & 1 & \vdots & 0 & \vdots & 0 & \vdots & 0 & \mid & 2 \\
\vdots & \vdots & \ddots & \vdots & \vdots & \vdots & \vdots & \vdots & \mid & \vdots \\
0 & 0 & \vdots & 1 & \vdots & 0 & \vdots & 0 & \mid & I \\
\vdots & \vdots & \vdots & \vdots & \ddots & \vdots & \vdots & \vdots & \mid & \vdots \\
0 & 0 & \vdots & 1 & \vdots & 0 & \vdots & 0 & \mid & J \\
\vdots & \vdots & \vdots & \vdots & \vdots & \vdots & \ddots & \cdots & \mid & \vdots \\
0 & 0 & \vdots & 0 & \vdots & 0 & . & 1 & \mid & n
\end{array}\right] .
$$

Figure 4 Boolean matrix $\left[T^{\Gamma}\right]$ for the condition when the value of the degree of freedom $J$ is constrained to equal the value of the degree of freedom 1 .

We also define $\Omega_{\text {domain_nodes }}$ as the set of all the nodes located in the domain. Thus, we can arrange all the components of the global characteristic vector $\{\chi\}^{k l}$, respecting the periodic boundary conditions as

$$
\{\boldsymbol{\chi}\}_{\Omega \text { domain_nodes }+\Gamma}=\left\{\begin{array}{c}
\{\boldsymbol{\chi}\}_{\Omega \text { domain_nodes }} \\
\{\boldsymbol{\chi}\}_{\Gamma}
\end{array}\right\}=\underbrace{\left[\begin{array}{cc}
{[\boldsymbol{I}]} & {[\mathbf{0}]} \\
{[\mathbf{0}]} & {\left[\boldsymbol{T}^{\Gamma}\right]}
\end{array}\right]}_{[\boldsymbol{T}]} \underbrace{\left\{\begin{array}{c}
\{\boldsymbol{\chi}\}_{\Omega \text { domain_nodes }} \\
\{\boldsymbol{\chi}\}_{\Gamma m}
\end{array}\right\}}_{\{\tilde{\boldsymbol{\chi}}\}}
$$

or

$$
\{\chi\}=[\boldsymbol{T}]\{\tilde{\chi}\}
$$

where $[\boldsymbol{T}]$ is the extended Boolean matrix comprising boundary and domain degrees of freedom.

Replacing Eq. (18) into Eq. (9) and pre-multiplying both sides by $[\boldsymbol{T}]^{T}$ leads to

$\left[\tilde{\boldsymbol{K}}^{A H}\right][\tilde{\chi}]=[\tilde{\boldsymbol{P}}]$, where

$\left[\tilde{\boldsymbol{K}}^{A H}\right]=[\boldsymbol{T}]^{T}\left[\boldsymbol{K}^{A H}\right][\boldsymbol{T}]$ and

$[\tilde{\boldsymbol{P}}]=[\boldsymbol{T}]^{T}[\boldsymbol{P}]$

For avoiding rigid body motion, it suffices to additionally constrain the displacements of one of the cell vertices to be zero. Due to periodicity all the vertices will also be constrained. After $\{\tilde{\chi}\}$ is determined, the whole set of 
characteristic displacements $[\chi]$ is recovered by means of Eq. (18). Once the characteristic displacements are calculated they are replaced in Eq. (5) or (6) to obtain the homogenized elastic tensor (and the corresponding compact notation matrix $[\boldsymbol{E}]^{H}$ ). In this method the homogenized tensor corresponds to an infinitesimal unit cell (a continuum point), which is not the same assumption to be considered for the EMsFEM. With this approach, the macrostructure can be modeled using a plane stress finite element, whose stiffness matrix is given by

$$
[\boldsymbol{k}]^{2 d}=\int_{-1}^{+1} \int_{-1}^{+1}[\boldsymbol{B}]^{T}[\boldsymbol{E}]^{H}[\boldsymbol{B}] J t d \xi d \eta
$$

where $[\boldsymbol{E}]^{H}$ is the constitutive matrix obtained via asymptotic homogenization, and $t=1$ since the width of the unit cell is given by the diameter of the thicker bar and is linked to the other cell dimensions. All the dimensions within the cell tend asymptotically to zero but keep the relative density constant by scaling proportionally the volume ocuppied by the bars and the total volume of the unit cell. In order to deal with this, we evaluate Eqs. (5) and (6) performing division by the area of the unit cell rather than the volume $|\mathrm{Y}|$, and making $t=1 \mathrm{in}$ Eq. (22). Hence, the numerical values in $[\boldsymbol{E}]^{H}$ must be adequately interpreted. For the evaluation of $[\boldsymbol{k}]^{2 d}$ we employ linear plane stress quadrilateral elements and integration is performed numerically by Gauss-Legendre quadrature using a $2 \times 2$ integration rule (full integration).

\section{EXTENDED MULTISCALE FINITE ELEMENT METHOD (EMsFEM)}

Hou and Wu (1996) proposed the Multiscale Finite Element Method (MSFEM) as a numerical tool for the solution of multiscale problems with reduced computational effort. The key idea relies on the use of numerically built discrete base (or interpolation) functions, which are defined on a finitely sized Representative Volume Element (RVE) or Unit Cell (UC). Therefore, the interpolation functions obtained incorporate automatically the influence of heterogeneities within the unit cell. Hence, the microscale information is introduced into the macroscale in the form of a stiffness matrix, which emulates the continuum, thus reducing drastically the number of degrees of freedom of the system to be solved. Zhang et al. (2009) used the idea of MSFEM to solve a coupled multiscale problem related to consolidation of saturated porous media, and called their method CMSFEM. In 2010 the same authors presented the Extended Multiscale Finite Element Method (EMsFEM), in which the construction of numerical interpolation functions take into account the coupled effects on displacements when applied to multidimensional problems (Zhang et al, 2010 a,b). The EMsFEM and AH share similarities but it is fundamental to remark that in the EMsFEM the macro/microscale ratio is finite while in AH it is not.

\subsection{Numerically built discrete interpolation functions}

The microscale structural response is accounted for using numerically built discrete interpolation functions, which must reproduce how the unit cell nodes respond to a unit displacement applied to each of its vertices in the directions of the global system of reference. This is achieved by defining a FEM problem to be solved at the unit cell level. The boundary conditions adopted in this problem deserve special discussion and are commented in deeper detail in subsections 3.1.1 ad 3.1.2. The system to be solved is

$$
\begin{aligned}
& {[\boldsymbol{K}]\{\boldsymbol{u}\}=\{\boldsymbol{F}\}} \\
& u_{\Gamma}=\bar{u}_{\Gamma}
\end{aligned}
$$

where the matrix $[\boldsymbol{K}]$ is formed by summing up the stiffness contribution of each bar within an arbitrary cell of the macroscopic structure, as shown in Fig. 5. A generic cellular structure including macro and microscales is presented in Fig. 5 (a) and the cell domain corresponding to Eq. (23) is depicted in the detail 5(b). Different boundary conditions can be considered to solve the finite element problem defined in the cell (Peric et al., 2011; Otero et al., 2018). In this work we compare two alternatives: linear (Hou and Wu, 1996; Zhang et al., 2010a) and periodic (Xia et al., 2006; Zhang et al., 2010b; Liu and Lv, 2017). The external force vector is set to zero. The nodal displacements $\{\boldsymbol{u}\}$ provide the numerically built discrete interpolation functions, corresponding to Fig. 6 or 7, for instance. Once we obtain the interpolation functions for the four vertices, we can develop the stiffness matrix $\left[\boldsymbol{K}_{E}\right]$ (of order $8 \times 8$ ) of the equivalent EMsFEM homogenized finite element, as shown in Fig. 5 (c). This stiffness matrix is then used to model the same structure of Fig. 5 (a). Clearly the dimension of the problem in Fig 5 (d) can be considerably smaller than the corresponding problem in 
Fig. 5 (a). The derivation of the interpolation functions for the linear and periodic cases is given in Sections 3.1 .1 and 3.1.2. The procedure for finding the associated matrix $\left[\boldsymbol{K}_{E}\right]$ is given in Section 3.1.3.

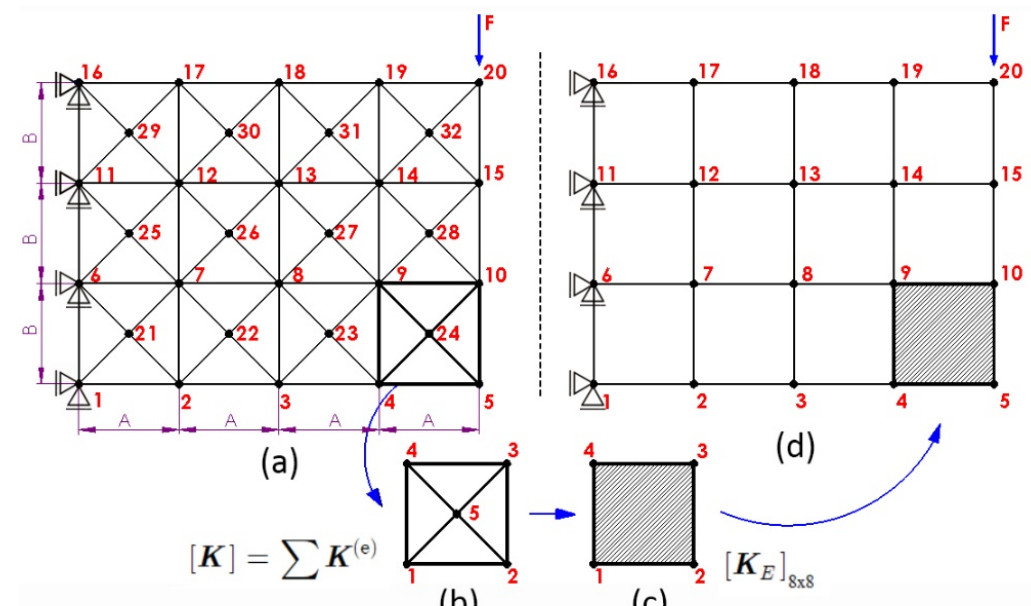

(b)

(c)

Figure 5 (a) generic cellular structure containing both macro and micro scales; (b) microscopic truss-like unit cell with its global stiffness matrix; (c) EMsFEM equivalent finite element; (d) macroscopic model with considerably less degrees of freedon compared to th model in (a).

\subsubsection{Linear boundary conditions for the EMsFEM}

Let the unit cell be the one defined in Fig. 6 by the counter clockwise vertices 1234. Following Zhang et al. (2010 a), we apply a unit displacement at node 1 in direction $x$ and impose a linear variation of this displacement from one to zero along sides 12 and 14 (Fig. 6b). The nodes on sides 23 and 34 are constrained to zero in the $x$ direction and all the border nodes are constrained to zero in $\mathrm{y}$. This problem is solved using the Finite Element Method and the nodal displacements provide a numerical (discrete) interpolation function associated to the first degree of freedom (node 1, direction $\mathrm{x}$ ). An analogous procedure is performed to obtain the numerical interpolation functions related to unit displacements prescribed in directions $x$ and $y$ for all the 4 vertices, hence making a total of 8 FEM problems. Notice that we must calculate the stiffness matrix just once since only the boundary conditions change. Nohtice also that all the borders remain straight, a constraint that is known to produce overstiffening, as already reported, in the context of $\mathrm{AH}$, by Sun and Vaidya (1996) and Xia et al. (2006), for instance. In our study we include this type of boundary condition to evaluate the extent of such overconstraining and for comparison with the results given by periodic boundary conditions. We refer to these boundary conditions as LBC.
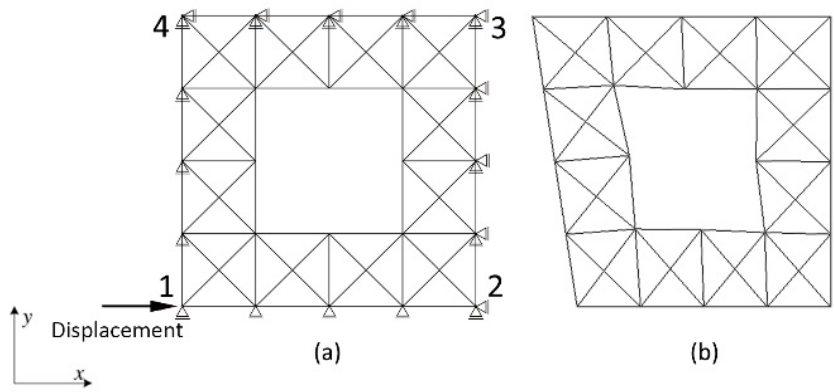

(b)

Figure 6 Scheme for construction EMsFEM using $L B C$.

\subsubsection{Periodic boundary conditions for the EMsFEM}

One remedy to overcome the overstiffening caused by linear boundary conditions (which enforce straight borders after deformation) is superposing a periodic displacement pattern. We will refer to these boundary conditions as PBC.

Let us consider the unit cell in Fig. 7, the boundary conditions associated to a unit horizontal displacement of node 1 are displayed in Fig. 7 (b). Such boundary conditions are composed of two parcels: a "linear boundary condition field $\{\overline{\Delta \boldsymbol{u}}\}$ ", in which the left and inferior edge suffer linearly varying displacements and the other edges are fixed (Fig. 7 (c)), 
and a "superposed periodic boundary condition field $\left\{\boldsymbol{u}_{p}\right\}$ ", in which the nodes on opposite edges must present the same displacements, as shown in Fig. 7 (d). Displacements in Fig. 7 (d) are amplified for visual clearness. The compliance of the cell in Fig. 7 (a) and (b) is augmented with respect to its LBC counterpart.

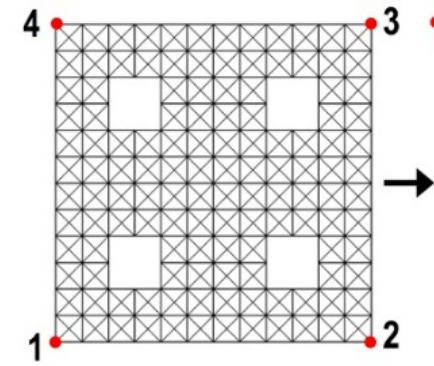

(a)

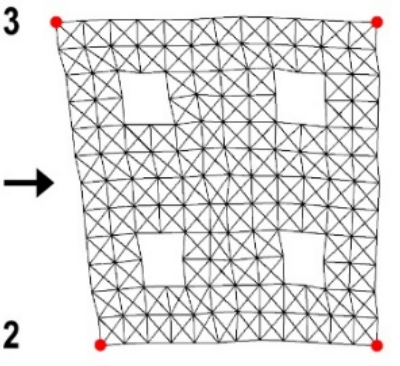

(b)

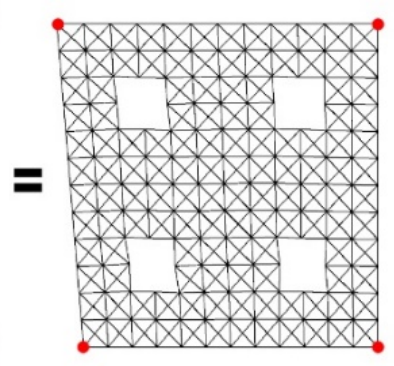

(c)

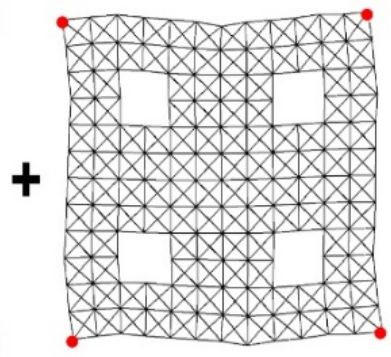

(d)

Figure 7 Numerical interpolation function obtained for the PBC scheme.

The interpolation functions now have, as boundary conditions, displacements that consist in the sum of the linear $\{\overline{\Delta \boldsymbol{u}}\}$ and periodic $\left\{\boldsymbol{u}_{p}\right\}$ parcels, and have the general form

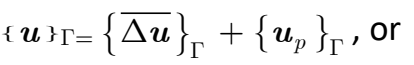

$\{\boldsymbol{u}\}_{\Gamma}=\left\{\begin{array}{c}\{\boldsymbol{u}\}_{\Gamma i} \\ \{\boldsymbol{u}\}_{\Gamma d}\end{array}\right\}=\left\{\begin{array}{l}\{\overline{\Delta \boldsymbol{u}}\}_{\Gamma i} \\ \{\boldsymbol{0}\}_{\Gamma d}\end{array}\right\}+\left\{\begin{array}{l}\left\{\boldsymbol{u}_{p}\right\}_{\Gamma i} \\ \left\{\boldsymbol{u}_{p}\right\}_{\Gamma d}\end{array}\right\}$

where $\{\boldsymbol{u}\}_{\Gamma},\{\overline{\Delta \boldsymbol{u}}\}_{\Gamma}$, and $\left\{\boldsymbol{u}_{p}\right\}_{\Gamma}$ are displacement vectors containing two degrees of freedom per node, $\Gamma$ is the set of nodes on the boundary, the bar indicates prescribed values and the subscript $p$ stands for "periodic" displacements.

The subscripts $i$ and $d$ stand for the faces that contain the independent and dependent degrees of freedom, respectively.

On the other hand, we have

$\left\{\begin{array}{l}\{\boldsymbol{u}\}_{\Gamma i} \\ \{\boldsymbol{u}\}_{\Gamma d}\end{array}\right\}=\left[\boldsymbol{T}^{\Gamma}\right]\left\{\boldsymbol{u}_{\Gamma i}-\left\{\begin{array}{l}\{\boldsymbol{0}\}_{\Gamma i} \\ \{\overline{\Delta \boldsymbol{u}}\}_{\Gamma d}\end{array}\right\}\right.$

where $\left[\boldsymbol{T}^{\Gamma}\right]$ is the same Boolean matrix shown in Section 2.1.1.

Replacing $\{\boldsymbol{u}\}_{\Gamma i}$ from Eq. (25) into Eq. (26), after some manipulations we get

$\{\boldsymbol{u}\}_{\Gamma}=\left\{\begin{array}{l}\{\boldsymbol{u}\}_{\Gamma i} \\ \{\boldsymbol{u}\}_{\Gamma d}\end{array}\right\}=\left[\boldsymbol{T}^{\Gamma}\right]\left\{\boldsymbol{u}_{p}\right\}_{\Gamma i}+\left\{\begin{array}{l}\{\overline{\Delta \boldsymbol{u}}\}_{\Gamma i} \\ {\left[\boldsymbol{T}_{d}\right]\{\overline{\Delta \boldsymbol{u}}\}_{\Gamma i}-\{\overline{\Delta \boldsymbol{u}}\}_{\Gamma d}}\end{array}\right\}$,

so that the global displacement vector related to the periodic boundary conditions is given by

$\{\boldsymbol{u}\}_{G}=\{\boldsymbol{u}\}_{\Omega+\Gamma}=\left\{\begin{array}{c}\{\boldsymbol{u}\}_{\Omega} \\ \{\boldsymbol{u}\}_{\Gamma i} \\ \{\boldsymbol{u}\}_{\Gamma d}\end{array}\right\}=\left[\begin{array}{cc}{[\boldsymbol{I}]} & {[\mathbf{0}]} \\ {[\mathbf{0}]} & {\left[\boldsymbol{T}^{\Gamma}\right]}\end{array}\right]\left\{\begin{array}{l}\{\boldsymbol{u}\}_{\Omega} \\ \{\boldsymbol{u}\}_{\Gamma i}\end{array}\right\}-\left\{\begin{array}{l}\{\mathbf{0}\}_{\Omega} \\ \{\overline{\Delta \boldsymbol{u}}\}_{\Gamma i} \\ {\left[\boldsymbol{T}_{d}\right]\{\overline{\Delta \boldsymbol{u}}\}_{\Gamma i}-\{\overline{\Delta \boldsymbol{u}}\}_{\Gamma d}}\end{array}\right\}$, or

$\{\boldsymbol{u}\}_{G}=[\boldsymbol{T}]\left\{\boldsymbol{u}_{R}\right\}_{\Omega+\Gamma i}+\{\overline{\Delta \boldsymbol{u}}\}_{\Omega+\Gamma}$ 
where $\left\{\boldsymbol{u}_{R}\right\}_{\Omega+\Gamma i}$ contains the domain degrees of freedom and the periodic part of the independent boundary degrees of freedom.

Replacing Eq. (29) into Eq. (23) and premultiplying the system by $[\boldsymbol{T}]^{T}$ we obtain the reduced system

$$
\begin{aligned}
& {\left[\boldsymbol{K}_{R}\right]\left\{\boldsymbol{u}_{R}\right\}_{\Omega+\Gamma i}=\left\{\boldsymbol{F}_{R}\right\} \text {, where }} \\
& {\left[\boldsymbol{K}_{R}\right]=[\boldsymbol{T}]^{T}[\boldsymbol{K}][\boldsymbol{T}] \text { and }\left\{\boldsymbol{F}_{R}\right\}=-[\boldsymbol{T}]^{T}[\boldsymbol{K}]\{\overline{\Delta \boldsymbol{u}}\}_{\Omega+\Gamma} .}
\end{aligned}
$$

\subsubsection{Numerical interpolation functions matrix and homogenized stiffness matrix}

According to the choice of boundary conditions LBC or PBC, and solving the system given by Eq. (23), we obtain all the nodal displacements of the discretized cell. This is done for each of the 8 cases of unit displacements ( 4 vertices and 2 degrees of freedom). We store all the displacements obtained in the vectors

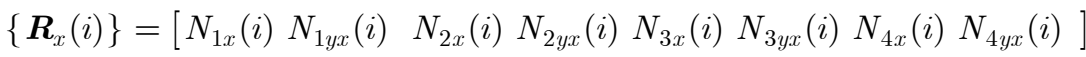

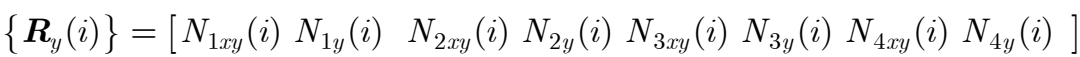

where the quantity stored in $N_{1 y x}(i)$ is the displacement of node i in the direction y, caused by a unit displacement applied to node 1 in direction $\mathrm{x}$. Therefore, the interpolation function matrix $[N]$ can be formed as

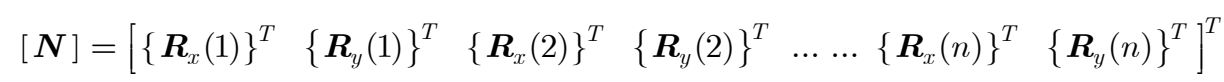

where $n$ is the total number of nodes of the fine-scale mesh within a unit cell.

Consider the arbitrary $e$-th bar element of the unit cell depicted in Fig. 8. The bar has two nodes, $p$ and $q$. For the element $e$, the strain energy can be evaluated by

$$
\pi^{(\mathrm{e})}=\frac{1}{2} k^{(\mathrm{e})}\left(\Delta L^{(\mathrm{e})}\right)^{2}
$$

where

$$
\Delta L^{(\mathrm{e})}=\left[\begin{array}{llll}
-\cos \theta^{(\mathrm{e})} & -\sin \theta^{(\mathrm{e})} & \cos \theta^{(\mathrm{e})} & \sin \theta^{(\mathrm{e})}
\end{array}\right]\left[\begin{array}{c}
u_{p} \\
v_{p} \\
u_{q} \\
v_{q}
\end{array}\right\} \text { and } k^{(\mathrm{e})}=\frac{E^{(\mathrm{e})} A^{(\mathrm{e})}}{L^{\mathrm{e})}}
$$

where $E$ is the Young modulus, $A$ is the cross section area and $L$ is the element length.

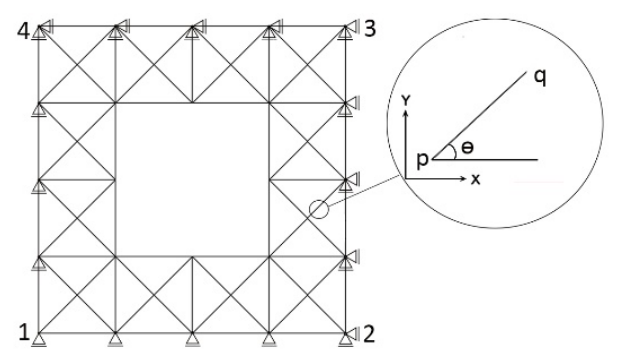

Figure 8 A particular element " $e$ " connecting nodes " $p$ " and " $q$ ".

The macroscale (vertex) displacements $\left\{\boldsymbol{u}_{E}^{\prime}\right\}$ are related to the microscale displacements $\left\{\boldsymbol{u}^{(\mathrm{e})}\right\}$ by the interpolation functions matrix, 
$\left\{\boldsymbol{u}^{(\mathrm{e})}\right\}=[\boldsymbol{N}]\left\{\boldsymbol{u}_{E}^{\prime}\right\}$

Using Eqs. 34 and 38 we obtain

$$
\left\{\begin{array}{c}
u_{p} \\
v_{p} \\
u_{q} \\
v_{q}
\end{array}\right\}=\left[\begin{array}{c}
R_{x}(p) \\
R_{y}(p) \\
R_{x}(q) \\
R_{y}(q)
\end{array}\right]\left\{\boldsymbol{u}_{E}^{\prime}\right\} .
$$

Further, using Eq. (36) and (39) it turns out that

$\Delta L^{(\mathrm{e})}=\left[\boldsymbol{G}^{(\mathrm{e})}\right]\left\{\boldsymbol{u}_{E}^{\prime}\right\}$, where

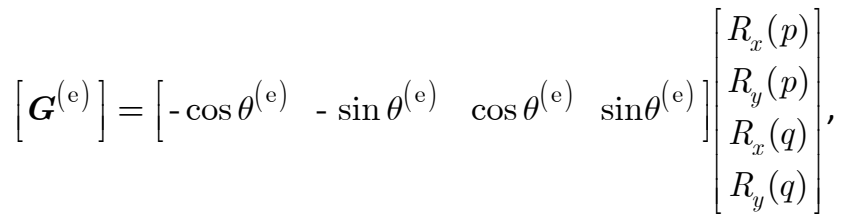

and introducing Eq. (36) into (37) we get the element strain energy expression

$$
\pi^{(\mathrm{e})}=\frac{1}{2}\left\{\boldsymbol{u}_{E}^{\prime}\right\}^{T}[\boldsymbol{G}]^{T} k^{(\mathrm{e})}\left[\boldsymbol{G}^{(\mathrm{e})}\right]\left\{\boldsymbol{u}_{E}^{\prime}\right\}
$$

Adding up the strain energy of all the bar elements in the cell, the total strain energy is

$\pi_{E}=\sum_{e=1}^{M} \pi^{(\mathrm{e})}=\frac{1}{2}\left\{\boldsymbol{u}_{E}^{\prime}\right\}^{T}\left[\sum_{e=1}^{M}\left[\boldsymbol{G}^{(\mathrm{e})}\right]^{T} k^{(\mathrm{e})}\left[\boldsymbol{G}^{(\mathrm{e})}\right]\right]\left\{\boldsymbol{u}_{E}^{\prime}\right\}$

where $M$ is the total number of elements within the unit cell.

From Eq. (43) we extract the cell homogenized stiffness matrix

$$
\left[\boldsymbol{K}_{E}\right]=\sum_{\mathrm{e}=1}^{M}\left[\boldsymbol{G}^{(\mathrm{e})}\right]^{T} k^{(\mathrm{e})}\left[\boldsymbol{G}^{(\mathrm{e})}\right]
$$

to be used in a finite element analysis to model the macroscale. The heterogeneities present at the microscale are incorporated in the cell $\left[\boldsymbol{K}_{E}\right]$, resulting in a direct linkage between macro and microscales. Notice that in contrast to AH, we do not deal with a homogenized material point, so there are no macroscopic strains or stresses, only macroscopic displacements. If stress and strain values are necessary, they are computed at the element level, whose displacements are obtained using Eq. (38).

Remark

In all the examples studied in this work, we explicitly show the interpolation functions obtained for linear and periodic boundary conditions as well as the corresponding stiffness matrices (in the Appendix 1). The purpose is twofold:

(i) on one hand we want to emphasize that linear boundary conditions always render overstiff unit cells, which in some cases might show a locking numerical pitfall; 
(ii) on the other hand we want to remark that especially for metamaterials, which are tailored for a given behavior, internal nodes might suffer spurious ${ }^{1}$ large displacements and deformations even for small displacements of the cell vertices. This happens in a number of examples trated in this study, which were verified against singularities by evaluation of the stiffness matrix eigenvalues. This issue requires attention because in such cases results are shown not to be trustfull.

\section{GUTH'S PERIODIC TRUSS METAMATERIALS}

All the unit cells studied here were borrowed from Guth etal. (2012), who employed sequential quadratic programming (SQP) (Schittkowski, 2006) and AH theory to obtain PTMs optimally tailored for extreme Poisson ratios. Guth took as initial design a symmetric square unit cell formed by truss elements with Young modulus and cross section areas given by $E=210[\mathrm{GPa}]$ and $A=10^{-5}\left[\mathrm{~m}^{2}\right]$, respectively. The length of the square unit cell adopted was equal to $0.1[\mathrm{~m}]$. The unit cell, the metamaterial and the homogenized elastic tensor $\left[\boldsymbol{E}^{H}\right]$ obtained by asymptotic homogenization are shown in Fig. 9. The Poisson ratios obtained by Guth use the relation

$\nu=\frac{E_{1122}^{H}}{E_{1111}^{H}}$

Notice that the numerical value of the components of $\left[\boldsymbol{E}^{H}\right]$ must be interpreted according to the discussion in Section 2.1.1. In order to display the tensor components per unit volume, the values presented in the forthcoming figures should be divided by the diameter of the thicker bar in the unit cell. As it is not possible to include the complete data for all the cells analyzed in this article, interested readers wishing to reproduce results can contact the authors.

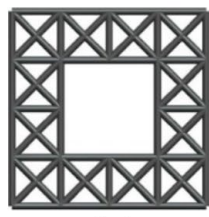

(a)

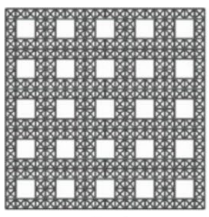

(b)

$$
\boldsymbol{E}^{\boldsymbol{H}}=\left[\begin{array}{ccc}
0.1150 & 0.02706 & 0 \\
0.02706 & 0.1150 & 0 \\
0 & 0 & 0.02405
\end{array}\right] \mathrm{GPa}
$$

(c)

Figure 9 Initial material design. (a) unit cell, (b) macroscopic material and (c) corresponding elastic homogenized tensor (Guth et al., 2012).

The homogenized elastic tensor $\left[\boldsymbol{E}^{H}\right]$ refers to an arbitrary point of a continuum homogeneous material subjected to plane stress conditions, equivalent to the porous material shown in Fig. 9 (b). In order to have a visual representation of the anisotropy level corresponding to the homogenized elastic tensors, we employ normalized diagrams showing the effect of rotations on $E_{1111}^{H}$ and $E_{1212}^{H}$. The reference for normalization is the largest value of the tensorial component in a $360^{\circ}$ rotation, as displayed in Fig. 10.

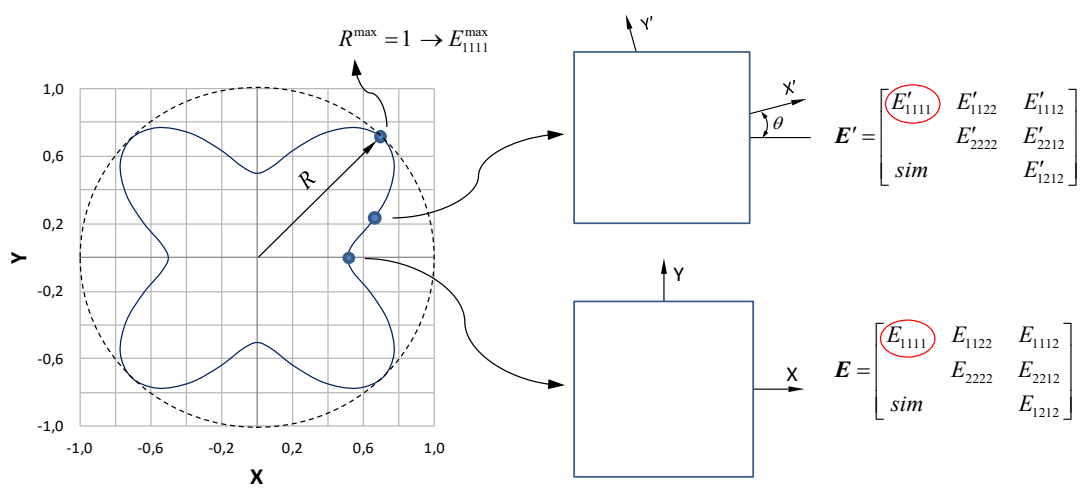

Figure 10 Polar plot of the component $E_{1111}^{H}$ rotated in the plane XY and normalized by $E_{1111}^{\max }$ (Guth et al., 2015).

\footnotetext{
${ }^{1}$ The term "spurious" here is used in the context that such displacements have no relation with the physical pattern obtained in the full micro-macroscale model.
} 
Analyzing $\left[\boldsymbol{E}^{H}\right]$ and the polar plots in Fig. 11, we can see that the obtained homogenized material shows orthotropic behavior. The optimization for maximum and minimum Poisson ratios were performed under two constraints: (i) mechanical isotropy and (ii) a constant value for the total volume occupied by the bars.

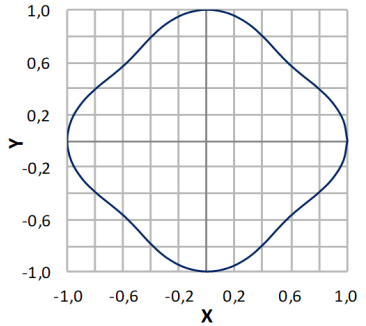

(a)

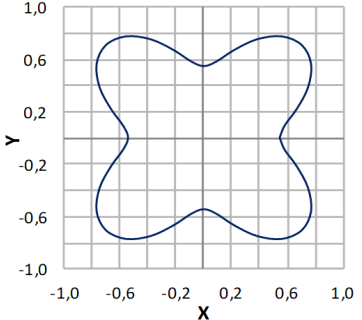

(b)

Figure 11 Polar plots for the elastic tensor (Guth 2012): (a) $E_{1111}^{H}$ component rotated and normalized with respect to $E_{1111}^{H}=0.1150 G P a ;(b) E_{1212}^{H}$ component rotated and normalized with respect to $\mathrm{E}_{1212}^{H}=0.0440 G P a$.

Noteworthy, in Guth unit cells the diagonal bars ovelap. Although not physically realistic, this type of modeling is frequent in truss topology optimization. We keep diagonals overlapping so that Guth's results can be adopted as references. A recent truss topology optimization work that prevents overlapping can be found in Cui and Huang (2018).

\subsection{Discussion on Guth's optimum unit cells for extreme Poisson ratios}

Guth's studies consider the traditional asymptotic homogenization procedure, which we described briefly in Section 2. In this method, the cells are subjected to imposed strain fields under periodic boundary conditions. Hence, the displacements suffered are always under control. In addition, the macroscopic (homogenized) properties do not take into consideration boundary effects, which are always present in pratical situations. Under these assumptions, Guth performed his optimization studies for maximum and minimum Poisson ratios taking as design variables: (i) only the elements cross section and (ii) the cross section and the nodal coordinates simultaneously.

Another issue of importance concerns the strategy to enforce isotropy in the opimum designs. The usual way to ensure isotropic behavior is, by construction, respecting a number of geometric symmetries of the unit cell. Following Neves et al. (2000), Guth did not enforce direct geometric conditions but relaxed this requirement and imposed isotropy by the introduction of a number of constraints in the optimization problem. This way, isotropy is not always satisfied exactly, but the domain of unit cells to be explored is enlarged and the isotropy violation in the final design is generally negligible for engineering purposes. Polar plots are included to emphasize that some unit cells studied are by no means intuitively isotropic, but the combination of their layout and spatial periodicity leads to a quasi-isotropic behavior, which can be considered isotropic for engineering purposes. Notwithstanding one has to consider that the isotropic behavior obtained by Guth corresponds to an infinitesimal unit cell and in the EMsFEM the microscale is finitely sized (as explained in Section 3). Hence, we perform numerical tests to evaluate if the application of EMsFEM (coarse mesh) to such asymmetric cells also leads to the same material behavior in different directions.

\subsubsection{Maximum Poisson ratio: only areas as design variables}

In this case, the optimum unit cell and the corresponding isotropic metamaterial are displayed in Figs. 12 (a) and (b). The Poisson ratio achieved is $\nu=0.99$ (the physical upper limit attainable for any 2D material is 1.0). The macroscopic (homogenized) elastic tensor is depicted in Fig. 12 (c), where the components are truncated at the fourth decimal digit. The polar plots in Fig. 13 show that the shear component is somewhat distant from the perfect circle that would characterize isotropy. However, the diagrams are nondimensional, and the normalizing value for the shear component is four orders of magnitude smaller than the axial counterpart. Therefore, the shear stiffness is comparatively negligible and the material can be considered isotropic for engineering purposes. 


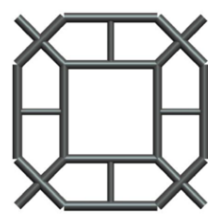

(a)

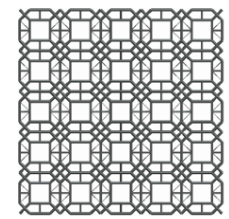

(b)

$$
\boldsymbol{E}^{\boldsymbol{H}}=\left[\begin{array}{ccc}
0.0739 & 0.0739 & 0 \\
0.0739 & 0.0739 & 0 \\
0 & 0 & 0
\end{array}\right] \mathrm{GPa}
$$

(c)

Figure 12 Initial material design. (a) unit cell, (b) macroscopic material and (c) corresponding elastic homogenized tensor (Guth 2012).

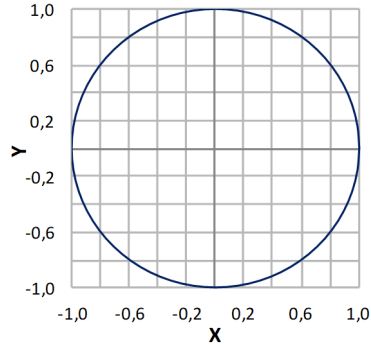

(a)

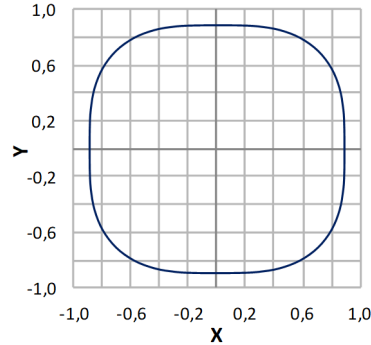

(b)

Figure 13 Polar plots for the elastic tensor (Guth 2012): (a) $E_{1111}^{H}$ component rotated and normalized with respect to $E_{1111}^{H}=0.0739 G P a ;(b) E_{1212}^{H}$ component rotated and normalized with respect to $E_{1212}^{H}=0.00001 G P a$.

4.1.2 Maximum Poisson ratio: simultaneous adoption of areas and nodal coordinates as design variables

In this case, the result for the optimum unit cell is shown in Fig. 14. The Poisson ratio of the isotropic metamaterial obtained is the same as in the previous case, $\nu=0.99$. The truncated homogenized elastic tensor is displayed in Fig. 14 (c). Notice that this unit cell layout is far from being intuitively isotropic. Notwithstanding, the polar plots in Fig. 15 confirm isotropy.

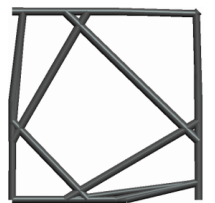

(a)

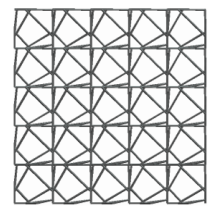

(b)

$$
\boldsymbol{E}^{\boldsymbol{H}}=\left[\begin{array}{ccc}
0.0537 & 0.0537 & 0 \\
0.0537 & 0.0537 & 0 \\
0 & 0 & 0
\end{array}\right] \quad \mathrm{GPa}
$$

(c)

Figure 14 Initial material design. (a) unit cell, (b) macroscopic material and (c) corresponding elastic homogenized tensor (Guth et al., 2012).

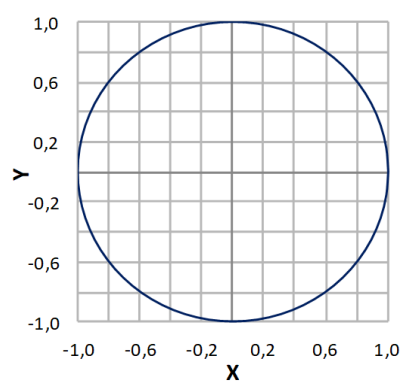

(a)

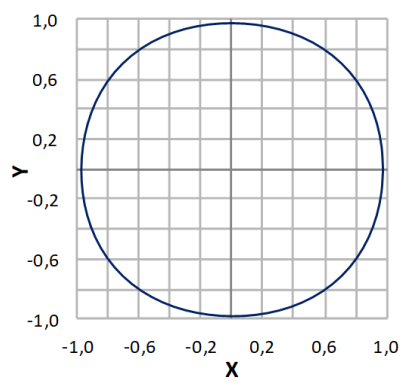

(b)

Figure 15 Polar plots for the elastic tensor (Guth 2012): (a) $E_{1111}^{H}$ component rotated and normalized with respect to $E_{1111}^{H}=0.0537 G P a ;(b) E_{1212}^{H}$ component rotated and normalized with respect to $E_{1212}^{H}=0.000004 G P a$. 


\subsubsection{Minimum Poisson ratio: only areas as design variables}

This cell was obtained in Guth's work aiming at an extreme auxetic material. The cell was optimized from the initial layout shown in Fig. 9 considering only the cross section of the bars as design variables. Mechanical isotropy was enforced as an optimization constraint. The unit cell obtained is displayed in Figs. 16 (a), the macroscopic material in Fig. 16 (b) and the homogenized constitutive tensor obtained via $\mathrm{AH}$ in Fig. 16 (c). Although the unusual cell layout precludes an intuitive perception of isotropy, the polar plots in Fig. 17 confirm this behavior. Different from the cases 4.2 and 4.3 , which aimed at Poisson ratio maximization, in this case the axial and shear homogenized components have the same order of magnitude. Hence, this material is able to withstand efficiently both, axial and shear loading. The Poisson ratio predicted by $\mathrm{AH}$ is $\nu=-0.99$. The minimum theoretical value attainable for $2 \mathrm{D}$ materials is $\nu=-1$.

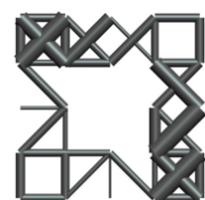

(a)

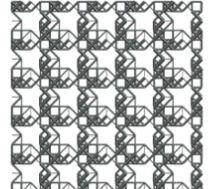

(a) (b)

Figure 16 Initial material design. (a) unit cell and (b) macroscopic material, (c) corresponding elastic homogenized tensor (Guth 2012).

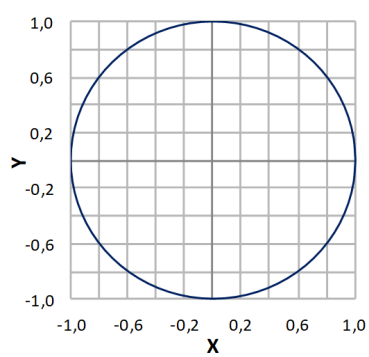

(a)

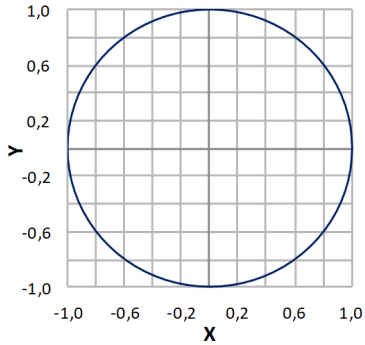

(b)

Figure 17 Polar plots for the elastic tensor (Guth 2012): (a) $E_{1111}^{H}$ component rotated and normalized with respect to $E_{1111}^{H}=0.0080 G P a ;(b) E_{1212}^{H}$ component rotated and normalized with respect to $E_{1212}^{H}=0.0080 G P a$.

4.1.4 Minimum Poisson ratio: simultaneous adoption of areas and nodal coordinates as design variables

The PTM considered in this section was tailored for minimum Poisson ratio, as the one in section 4.1.3. In this case, however, both coordinates and cross section areas were adopted as design variables. The Poisson ratio achieved was $\nu=-0.99$. The optimization was constrained for mechanical isotropy but leaded to a non-symmertic unit cell which would hardly be visually interpreted as isotropic (Fig. 18a). The AH homogenized constitutive tensor is shown in Fig. 18 (c), and its isotropic nature can be is confirmed by the polar plots in Fig. 19. Similar to the case 4.1.3, the axial and shear homogenized components have the same order of magnitude. Thus, this material is able to support both, axial and shear loading.

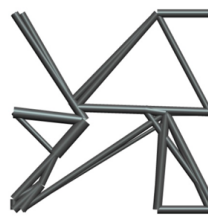

(a)

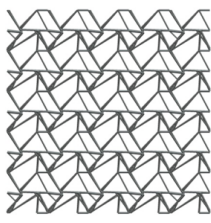

(b)

$$
\boldsymbol{E}^{\boldsymbol{H}}=\left[\begin{array}{ccc}
0.0119 & -0.0119 & 0 \\
-0.0119 & 0.0119 & 0 \\
0 & 0 & 0.0119
\end{array}\right] \mathrm{GPa}
$$

(c)

Figure 18 Initial material design. (a) unit cell, (b) macroscopic material and (c) corresponding elastic homogenized tensor (Guth 2012). 


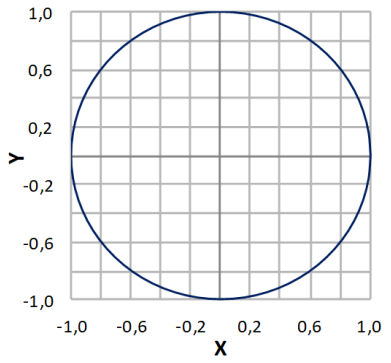

(a)

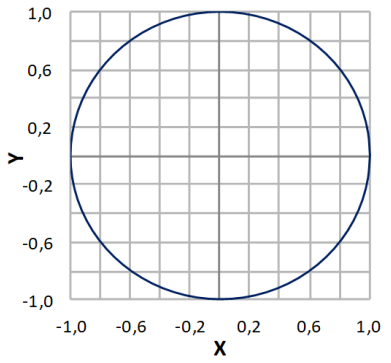

(b)

Figure 19 Polar plots for the elastic tensor (Guth 2012): (a) $E_{1111}^{H}$ component rotated and normalized with respect to $E_{1111}^{H}=0.0120 G P a ;(b) E_{1212}^{H}$ component rotated and normalized with respect to $E_{1212}^{H}=0.0120 G P a$.

\section{DETERMINATION OF EMSFEM INTERPOLATION FUNCTIONS FOR GUTH'S PERIODIC TRUSS METAMATERIALS}

In this section we show and discuss the numerical interpolation functions obtained for each unit cell using LBC and PBC boundary conditions. While the lower compliance produced by the LBC (when compared to PBC) is visually observed in the Figures for all the cases, this effect is displayed quantitatively in the corresponding stiffness matrices presented in the Appendix 1. The overstiffening produced by LBC has a strong influence in modeling macroscopic components, in some cases leading to numerical locking, as reported in Section 6. As a quantitative measure of stiffnening, we compute the percentual difference beween the LBC and PBC stiffness matrices measured by the Frobenius norm, i.e.,

$$
E_{K}=\frac{\left\|\boldsymbol{K}_{E}^{L B C}\right\|-\left\|\boldsymbol{K}_{E}^{P B C}\right\|}{\left\|\boldsymbol{K}_{E}^{P B C}\right\|} \times 100
$$

and

$$
\left\|K_{E}\right\|=\sqrt{\sum\left(K_{E i j}\right)^{2}}
$$

where $E_{K}$ is the ratio of the Frobenius norm between unit cells.

Special attention is given to the case 5.3, corresponding to the unit cell for maximum Poisson ratio with areas and coordinates as design variables. The interpolation functions of this particular unit cell display unexpected excessive displacements for nodes inside the cell. We suggest a possible explanation for this response and anticipate difficulties for modeling a macroscopic component with this cell.

\subsection{EMsFEM Interpolation functions for the initial cell}

The numerical interpolation functions obtained for the initial cell by application of linear and periodic boundary conditions are displayed in Figs. 20 and 21, respectively. The stiffness difference is not very significant, as confirmed by the numerical values of the corresponding homogenized stiffness matrices, in the Appendix 1 . The ratio of the Frobenius norm is $\left\|\boldsymbol{K}_{E}^{L B C}\right\| /\left\|\boldsymbol{K}_{E}^{P B C}\right\|=1.054$, that is, a percentage stiffening $E_{K}$ of only $5.4 \%$. As a consequence, the application of $\mathrm{LBC}$ or PBC do not to affect much the results for macroscopic problems modelled with the EMsFEM in Section 6.

\subsection{EMsFEM interpolation functions for the cell corresponding to maximum Poisson ratio - only areas}

The numerical interpolation functions obtained for this cell by application of linear and periodic boundary conditions are displayed in Figs. 22 and 23 evincing visually a larger stiffness associated to linear boundary conditions. The stiffness difference can be appreciated quantitatively in the Appendix 1, which shows the numerical values of the corresponding homogenized stiffness matrices. The stiffness difference can be quantified by the Frobenius norm, which provides a justification to the LBC overstiffening suffered in the macroscopic problem in Section 6 . In this case, the ratio of the Frobenius norm is $\left\|\boldsymbol{K}_{E}^{L B C}\right\| /\left\|\boldsymbol{K}_{E}^{P B C}\right\|=1.068$, that is, a small percentage stiffening $E_{K}=6.8 \%$. 


\subsection{EMsFEM interpolation functions for the cell corresponding to maximum Poisson ratio - areas and coordinates}

The numerical interpolation functions obtained for this cell by application of linear and periodic boundary conditions are displayed in Figs. 24 and 25. The larger stiffness associated to the linear boundary conditions can be visualized in the Figures and analyzed quantitavely in the homogenized stiffness matrices presented in the Appendix 1 . In this case, the ratio of the Frobenius norm is very significant $\left\|\boldsymbol{K}_{E}^{L B C}\right\| /\left\|\boldsymbol{K}_{E}^{P B C}\right\|=1.467$ or $E_{K}=46.7 \%$.

An explanation to the strange displacements reported in Figs. 24 and 25 is given in Fig. 26. As the unit cell of this PTMM has some bars disposed almost collinearly, a simple vector decomposition reveals that very large axial forces are exerted on them, causing large stresses and strains, resulting in excessive displacements. We remark that an eigenvalue analysis was performed on the stiffness matrices, excluding the possibility of singularity. It is important to realize that AH was not capable of detecting this numerical pitfall, as it only becomes apparent when considering a finite dimension of the microscale. On the other hand, the phenomenon is easily perceived in the EMsFEM approach by simple observation of the interpolation functions. Another issue to remark is that the unit cells obtained by Guth were optimized under the assumption of geometric linearity, so we also limit the EMsFEM analyses to this condition (in which large displacements are not allowed).
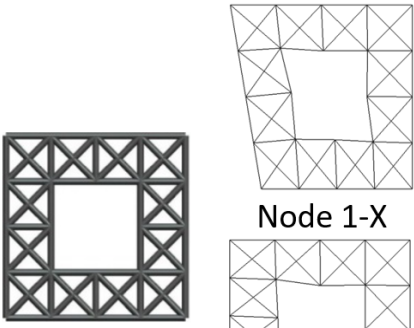

$$
\text { Node 1-X }
$$

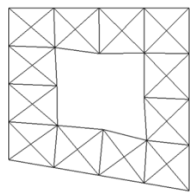

Node 1-Y

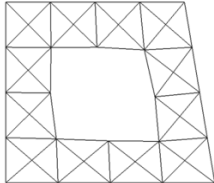

Node 2-X

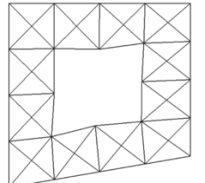

Node $2-Y$

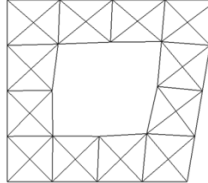

Node 3-X

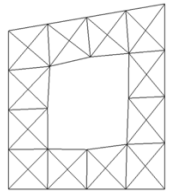

Node 3-Y

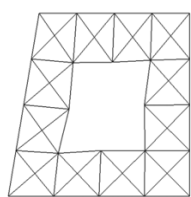

Node 4-X

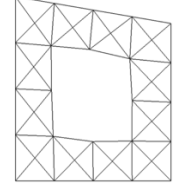

Node 4-Y

Figure $\mathbf{2 0}$ Numerical interpolation functions obtained for $L B C$.
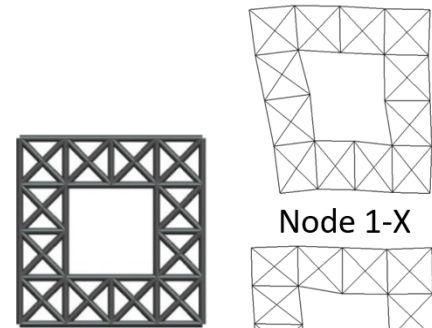

Node 1-X

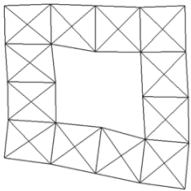

Node 1-Y

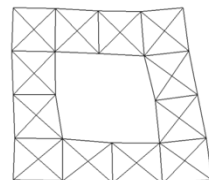

Node 2-X

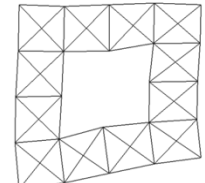

Node 2-Y

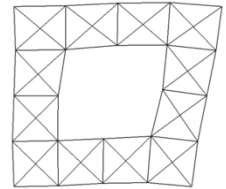

Node $3-X$

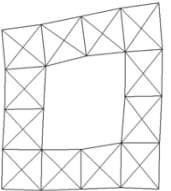

Node 3-Y

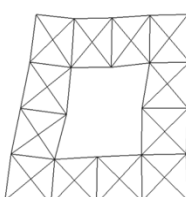

Node 4-X

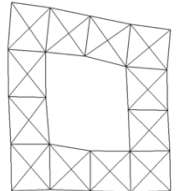

Node 4-Y

Figure 21 Numerical interpolation functions obtained for $P B C$

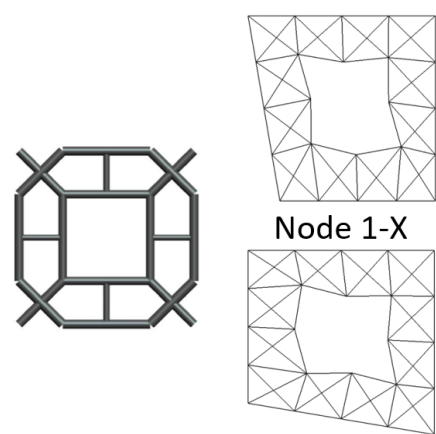

Node 1-Y

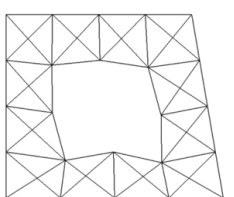

Node 2-X

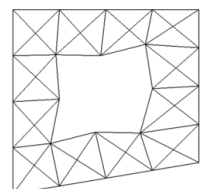

Node 2-Y

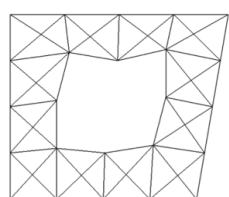

Node 3-X

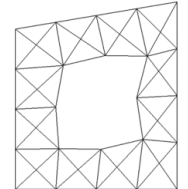

Node 3-Y

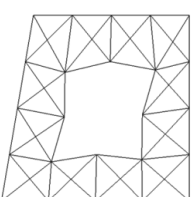

Node 4-X

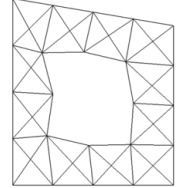

Node 4-Y

Figure 22 Numerical interpolation functions obtained for $L B C$. 

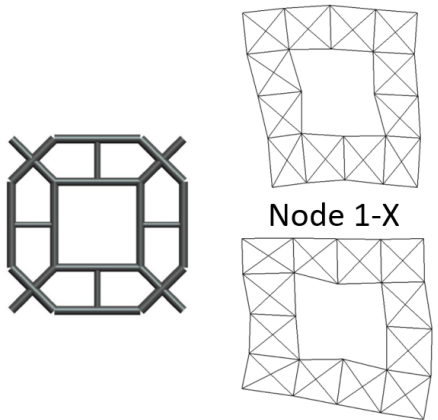

Node 1-Y

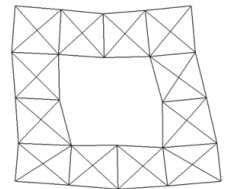

Node 2-X

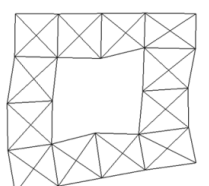

Node 2-Y

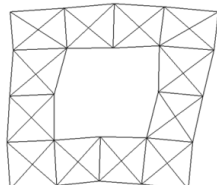

Node 3-X

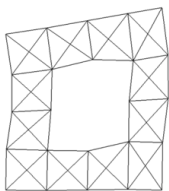

Node 3-Y

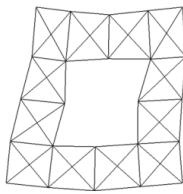

Node 4-X

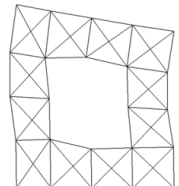

Node 4-Y

Figure 23 Numerical interpolation functions obtained for $P B C$.

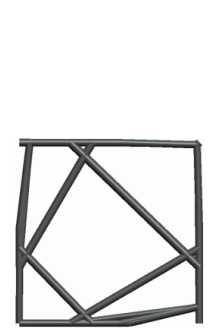

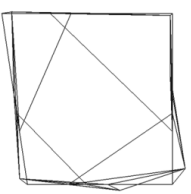

Node 1-X

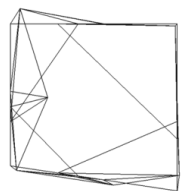

Node 1-Y

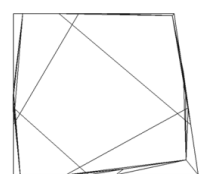

Node 2-X

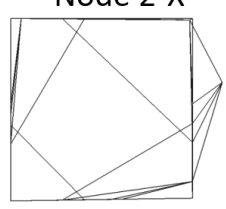

Node 2-Y

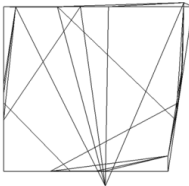

Node 3-X

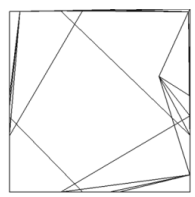

Node $3-Y$

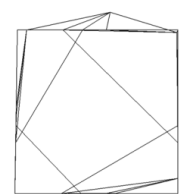

Node 4-X

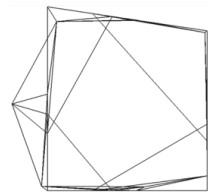

Node 4-Y

Figure 24 Numerical interpolation functions obtained for $L B C$
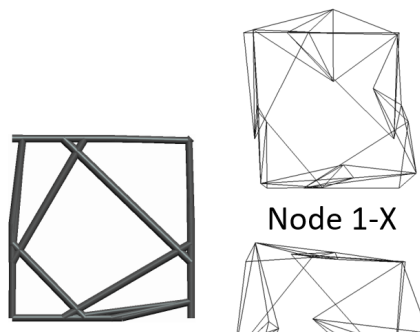

Node 1-X

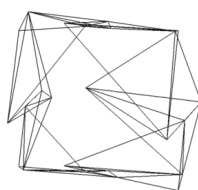

Node 1-Y

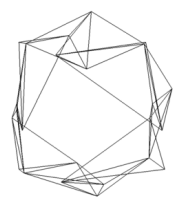

Node 2-X

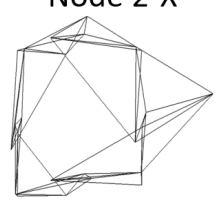

Node 2-Y

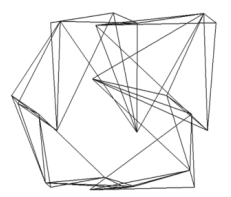

Node 3-X

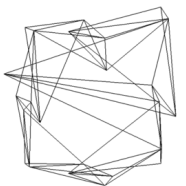

Node 3-Y

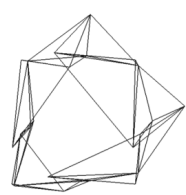

Node 4-X

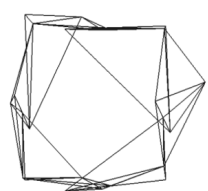

Node 4-Y

Figure 25 Numerical interpolation functions obtained for $P B C$.

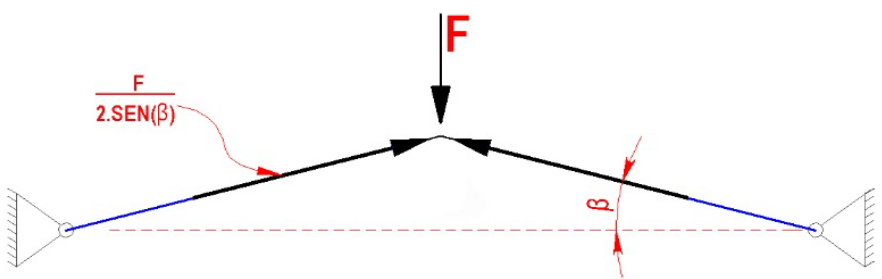

Figure 26 Force decomposition showing large axial resultants on nearly collinear bars.

\subsection{EMsFEM interpolation functions for the cell corresponding to minimum Poisson ratio - only areas}

The numerical interpolation functions obtained for this cell by application of linear and periodic boundary conditions are displayed in Figs. 27 and 28. The Appendix1 displays the corresponding stiffness matrices showing significant differences. The Frobenius norm of these matrices puts in evidence a severe numerical stiffening for linear boundary conditions, with a ratio $\left\|\boldsymbol{K}_{E}^{L B C}\right\| /\left\|\boldsymbol{K}_{E}^{P B C}\right\|=2.81$ or or $E_{K}=181 \%$. Accordingly, the macroscale problem studied in Section 6 shows that the EMsFEM-LBC fails at providing a good approximation for the full micro-macroscale model. 


\subsection{EMsFEM interpolation functions for the cell corresponding to minimum Poisson ratio - areas and coordinates}

The numerical interpolation functions obtained for this cell by application of linear and periodic boundary conditions are displayed in Figs. 29 and 30. The Appendix 1 displays the corresponding stiffness matrices showing that not only the magnitude of stiffness components differ considerably but their signal may change. The ratio of the Frobenius norm is $\left\|\boldsymbol{K}_{E}^{L B C}\right\| /\left\|\boldsymbol{K}_{E}^{P B C}\right\|=2.15$, corresponding to a percentual stiffening of $E_{K}=115 \%$. Implications for modelling macroscopic structures are foreseen. In fact, application of this cell in the macroscale problem studied in Section 6 evinces that the EMsFEM-LBC causes locking for vertical displacements.

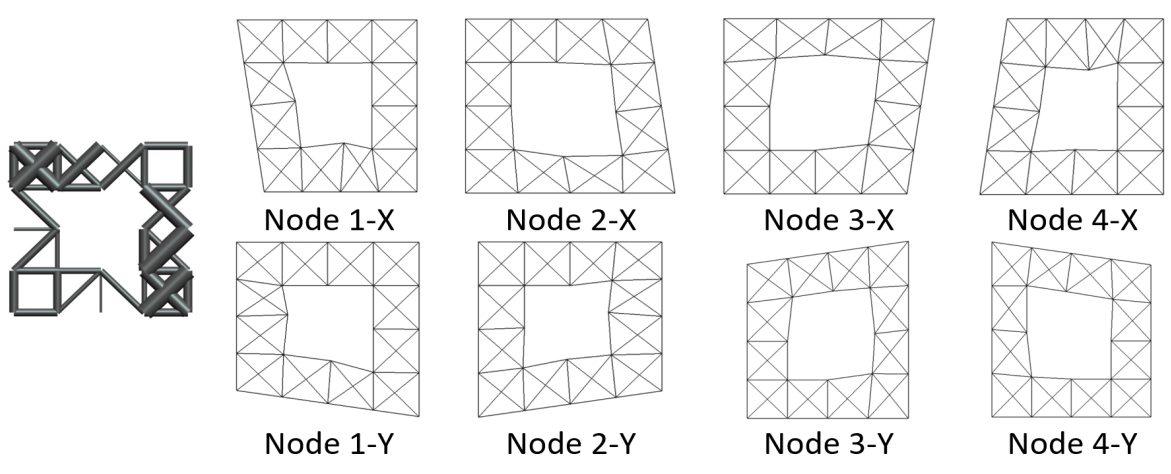

Figure 27 Numerical interpolation functions obtained for $L B C$
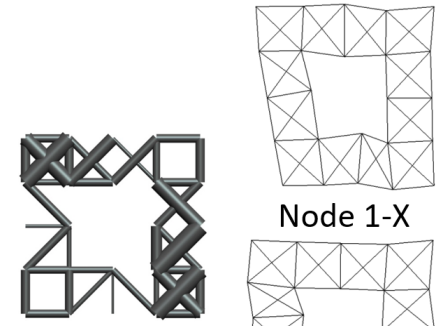

Node 1-X

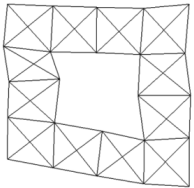

Node 1-Y

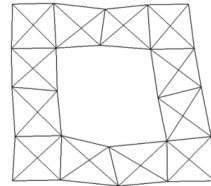

Node 2-X

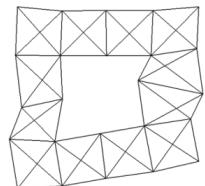

Node 2-Y

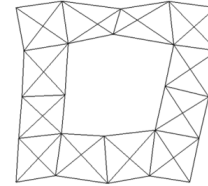

Node 3-X

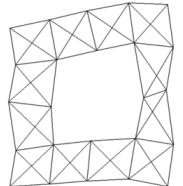

Node 3-Y

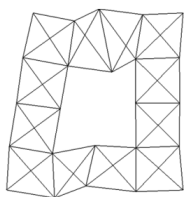

Node 4-X

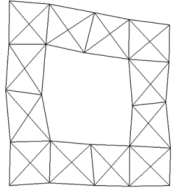

Node 4-Y

Figure 28 Numerical interpolation functions obtained for $P B C$.
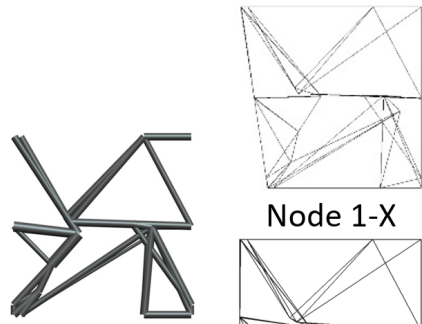

Node 1-X

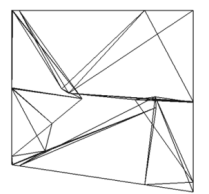

Node 1-Y

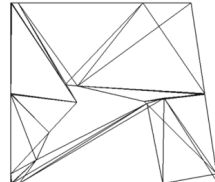

Node 2-X

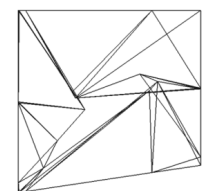

Node 2-Y

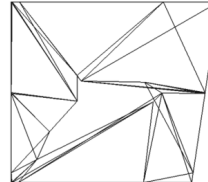

Node 3-X

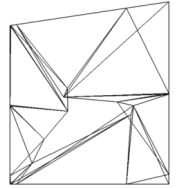

Node 3-Y

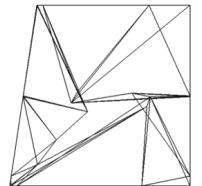

Node 4-X

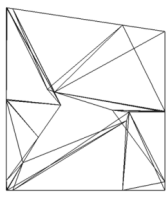

Node 4-Y

Figure 29 Numerical interpolation functions obtained for LBC. 

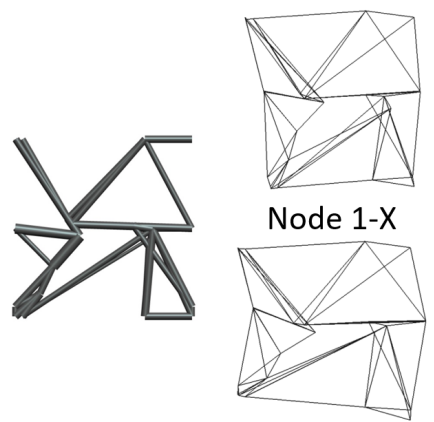

Node 1-Y

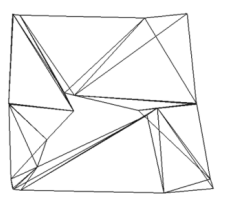

Node 2-X

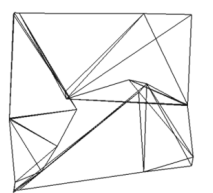

Node 2-Y

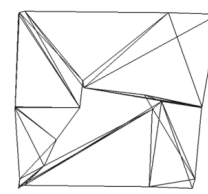

Node 3-X

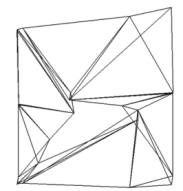

Node 3-Y

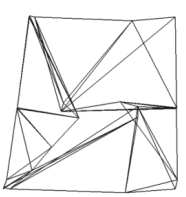

Node 4-X

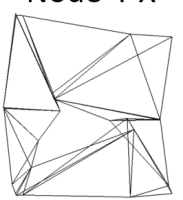

Node 4-Y

Figure 30 Numerical interpolation functions obtained for $P B C$.

\section{MACROSCOPIC EVALUATION OF THE PTMMS POISSON RATIO USING THE EMsFEM}

The EMsFEM is employed to evaluate the macroscopic behavior of a rectangular plate made up of PTMM cells. The dimension of the plate is $1 \times 0.6 \mathrm{~m}$ (length $\times$ height) and the FEM discretization is shown in Fig. 31 . Each finite element equals one unit cell. The plate is subjected to traction by prescribed uniform unit horizontal displacements $|u x|=0.1$ at both lateral edges. In order to evaluate the Poisson ratio for each of the unit cells depicted in the Figure, we calculate the horizontal/vertical displacement ratio in a selected subdomain inside the plate. We compare the results with the values predicted by asymptotic homogenization (Guth, 2012), emphasizing that due to to the finite dimension of the microscale, such results are not necessarily coincident. The Poisson ratio given by Guth is obtained by relations between the homogenized tensor components. We also verify the Poisson ratio introducing the homogenized constitutive tensors found by Guth (and presented in Section 5) in the plane stress FEM formulation given by Eq. (21). In this case we keep the same finite element size $\Delta$ used by the EMsFEM but the size of the unit cell tends to zero. The Poisson ratio obtained this way is given in the bottom of Figs. 32-36.

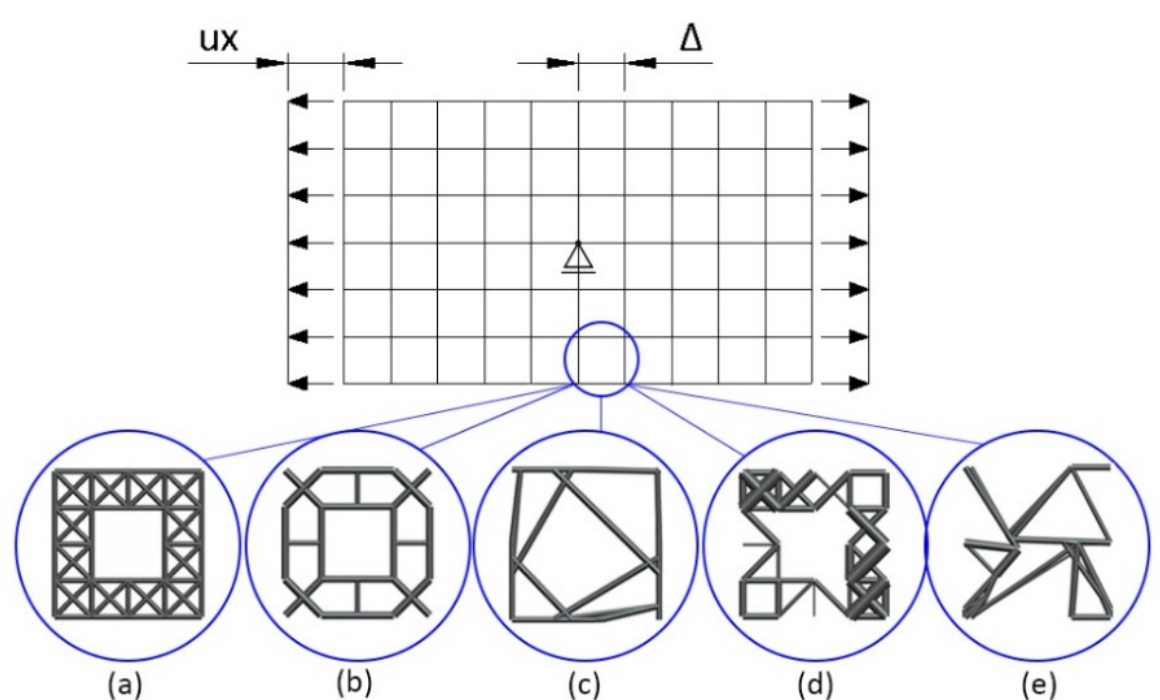

Figure 31 FEM discretization macro and micro scale problem.

\subsection{Initial cell}

The results for this cell are shown in Fig. 32. The real physical situation is given by the full-bar model, where the upper and lower plate edges are free to move. The Poisson ratio is estimated over the indicated area, resulting in $\nu=$ 0.2353 . The EMsFEM-LBC approximation presents a result of $\nu=0.2391$, whereas the value obtained using the EMsFEMPBC is $\nu=0.2353$, in exact agreement with the full bar model. Both EMsFEM alternatives provide good approximations 
for the displacement field pattern (isocolor map) and quantitative values. The use of a plane stress FEM model with the AH elastic tensor also leads to $\nu=0.2353$ and similar displacement distribution.

\subsection{Optimized cell for maximum Poisson ratio - area}

For this cell and objective, Guth's optimization considered only the element cross section areas as design variables, obtaining a nearly symmetrical layout. The AH prediction for an infinitesimal microscale indicates isotropic behavior with a Poisson ratio $\nu=0.99$. Figure 33 shows results for the finitely microscaled full bar model, whose Poisson ratio is evaluated as $\nu=0.9997$ over the depicted region. The EMsFEM-LBC and EMsFEM-PBC approximations provide $\nu=$ 0.5316 and $\nu=0.9998$, respectively. A significant overstiffening is observed for the LBC as foreseen in Section 5.2. Downscaling confirms a good agreement between the EMsFEM-PBC and the full bar model both in displamement distribution and values.

\subsection{Optimized cell for maximum Poisson ratio - area and coordinates}

This case, shown in Fig. 34, deserves special attention. The unit cell is asymmetrical, far from being intiuitively isotropic. Notwithstanding, for an infinitesimal microscale, the AH predicts isotropy and a Poisson ratio $\nu=0.99$. Evaluation of the Poisson ratio in the selected region of the full-bar domain results in $\nu=0.9978$. The EMsFEM-LBC shows overstiffening again (with $\nu=0.4979$ ) whereas the EMsFEM-PBC leads to a qualitatively fair agreement with the full bar model for locations apart from the borders $(\nu=0.9996)$. For this unit cell, spurious values are detected for downscaled EMsFEM-LBC displacements. Hence, with this EMsFEM alternative it is not possible to predict local failure nor to perform nonlinear analyses.

\subsection{Optimized cell for minimum Poisson ratio - area}

This example concerns the unit cell designed for mimimum Poisson ratio when only areas are adopted as design variables. For an infinitesimal cell with this layout, the AH performed by Guth predicts $\nu=-0.99$. However, due to size and border effects, Fig. 35 shows that result obtained for the full bar model (10 66 cells) is very different, $\nu=-0.677$ when evaluated over the highlighted region. Furthermore, EMsFEM-LBC provides a completely erroneous result dominated by a rigid rotation, leading to $\nu=-0.1720$. On the other hand, the Poisson ratio given by EMsFEM-PBC is $\nu=-0.9929$, and is in in closer accordance with AH than with the $10 \times 6$ full bar model. This probably happens because the kinematic periodic boundary conditions imposed in EMsFEM-PBC are able to reproduce better the AH periodic boundary conditions than the free edge displacements of the full model. So, if the goal is reproducing the full bar model none of the EMsFEM versions are satisfactory. In order to obtain a better insight into the problem we perform a similar analysis, this time containing $60 \times 30$ cells, keeping the same cell dimensions. These additional results are included in the Figure, where it is observed that far enough from the border, the Poisson ratio obtained is very close to -1 .

\subsection{Optimized cell for minimum Poisson ratio - area and coordinates}

In Fig. 36 we show the behavior of the material generated by the periodic repetition of Guth's unit cell designed for minimum Poisson ratio when both areas and coordinates are adopted as design variables. The Poisson ratio predicted by AH is $\nu=-0.99$, whereas the value calculated with basis on the selected region of the full bar model $(10 \times 6$ cells) is $\nu=-0.7443$. The EMsFEM should approach the full bar model but the results obtained for the EMsFEM-LBC and for the EMsFEM-PBC are $\nu=-0.0513$ and $\nu=-0.9968$, respectively. As in case 6.4, nor of the EMsFEM versions is satisfactory. The EMsFEM-LBC locks and the EMsFEMPBC tends to the AH solution rather than to the full bar model. Again, this fact stems from size and border effects. As for the cell in Fig. 35, a $60 \times 30$ cells structure is studied, keeping the cell size and the macroscopic proportions. For this mesh and far enough from the border, we verify that the Poisson ratio obtained is $\nu=-0.9986$. 


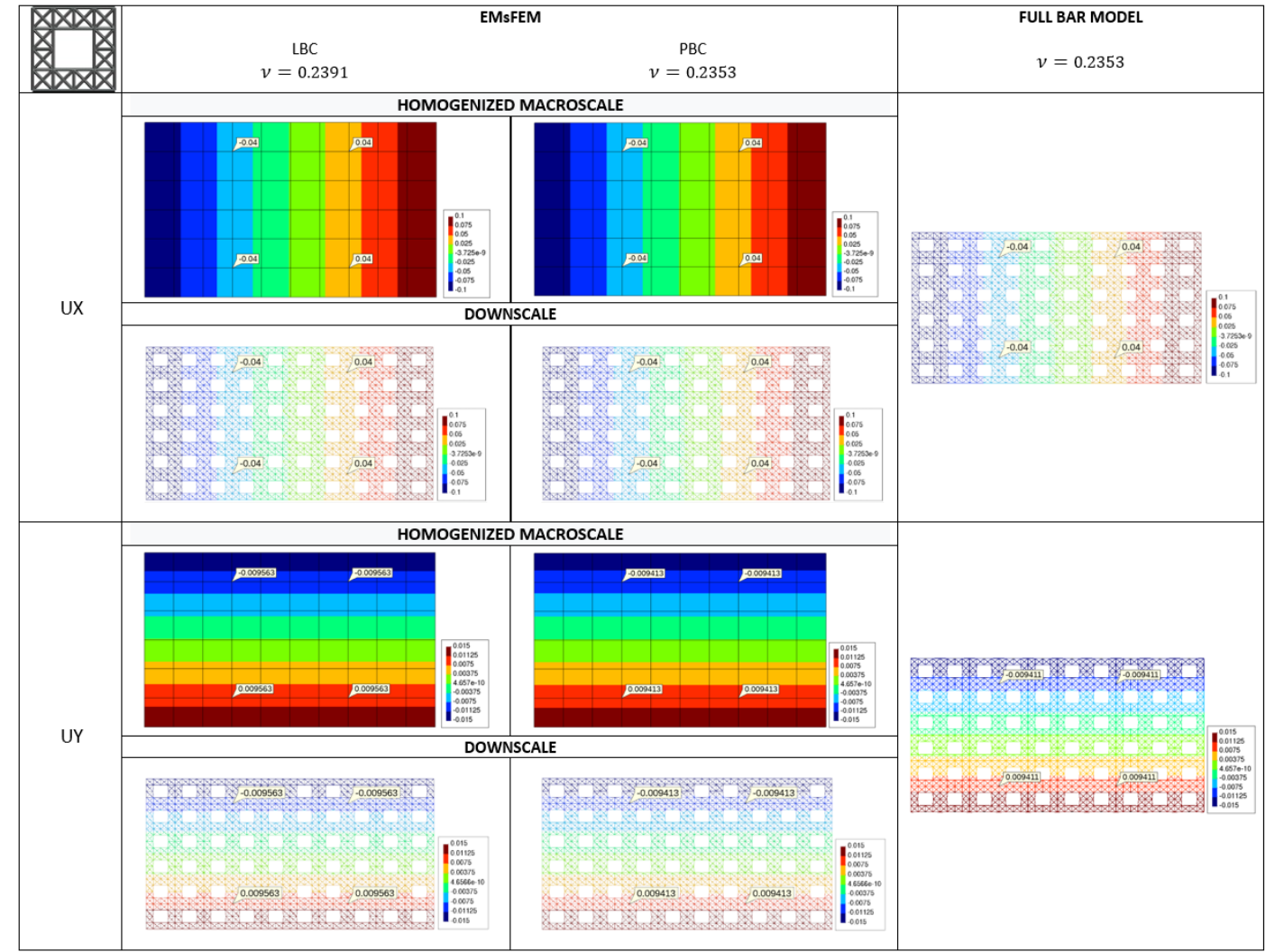

Figure 32 Poisson ratio evaluated using EMSFEM - initial cell plane stress FEM fully integrated linear quadrilateral element (AH) $v=0.23532$.

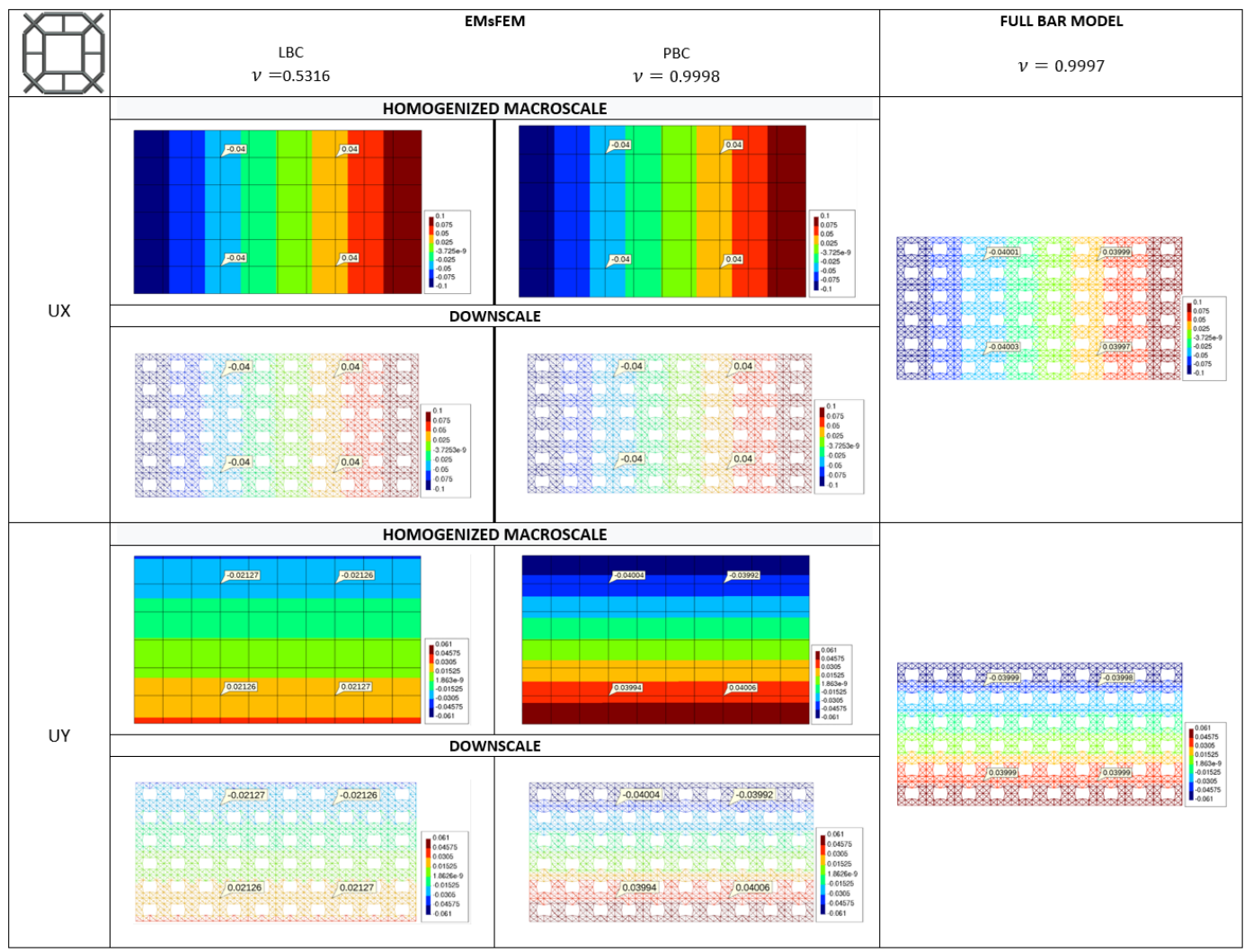

Figure 33 Max. Poisson ratio evaluated using EMSFEM - only areas I plane stress FEM fully integrated linear quadrilateral element $(\mathrm{AH})-v=0.99975$. 


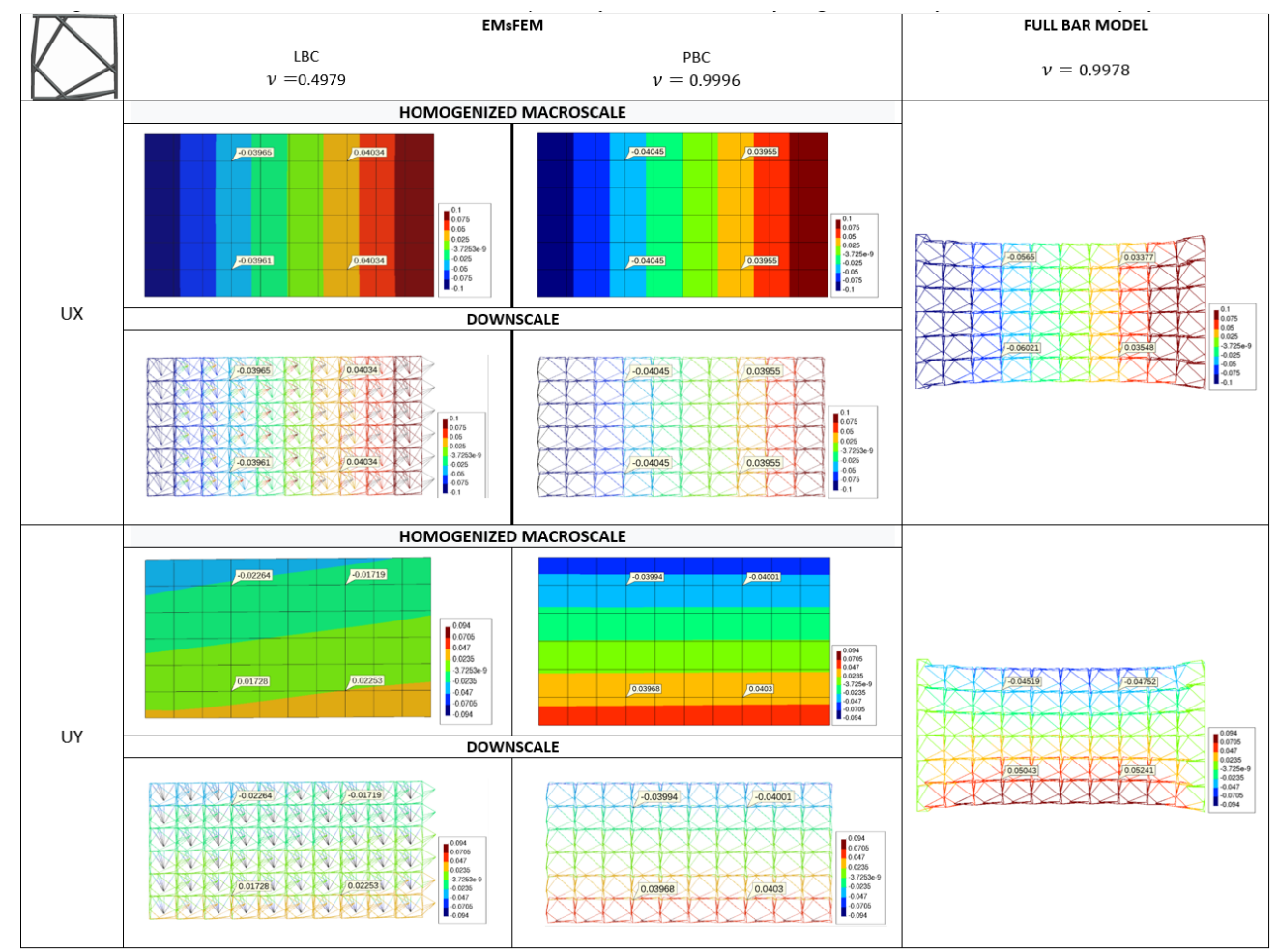

Figure 34 Max. Poisson ratio evaluated using EMSFEM - areas and coordinates plane stress FEM fully integrated linear quad. element $(A H)-v=0.99975$.

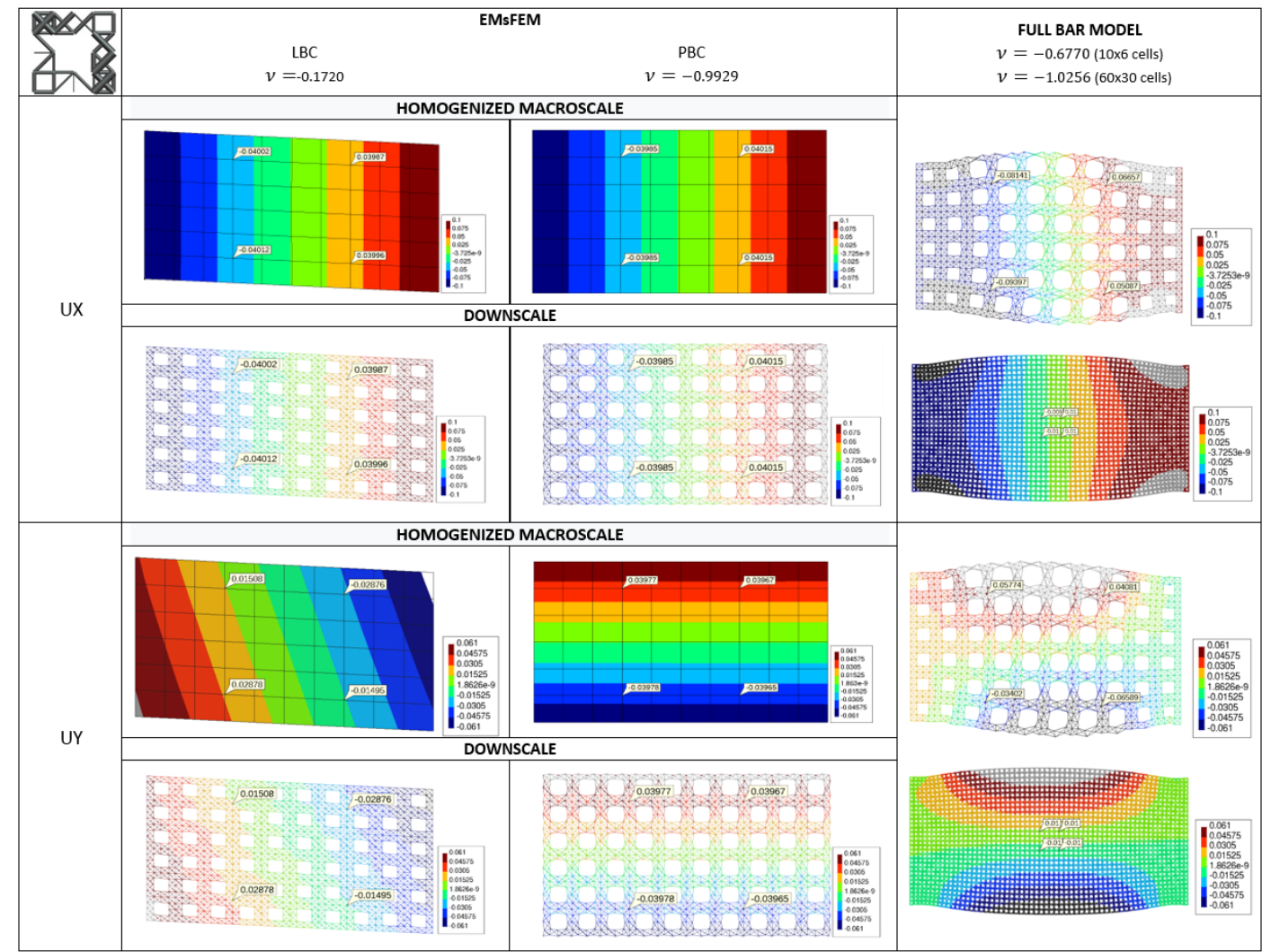

Figure 35 Min. Poisson ratio evaluated using EMsFEM - only areas plane stress FEM fully integrated linear quad. element (AH) $v=-0.9930$. 


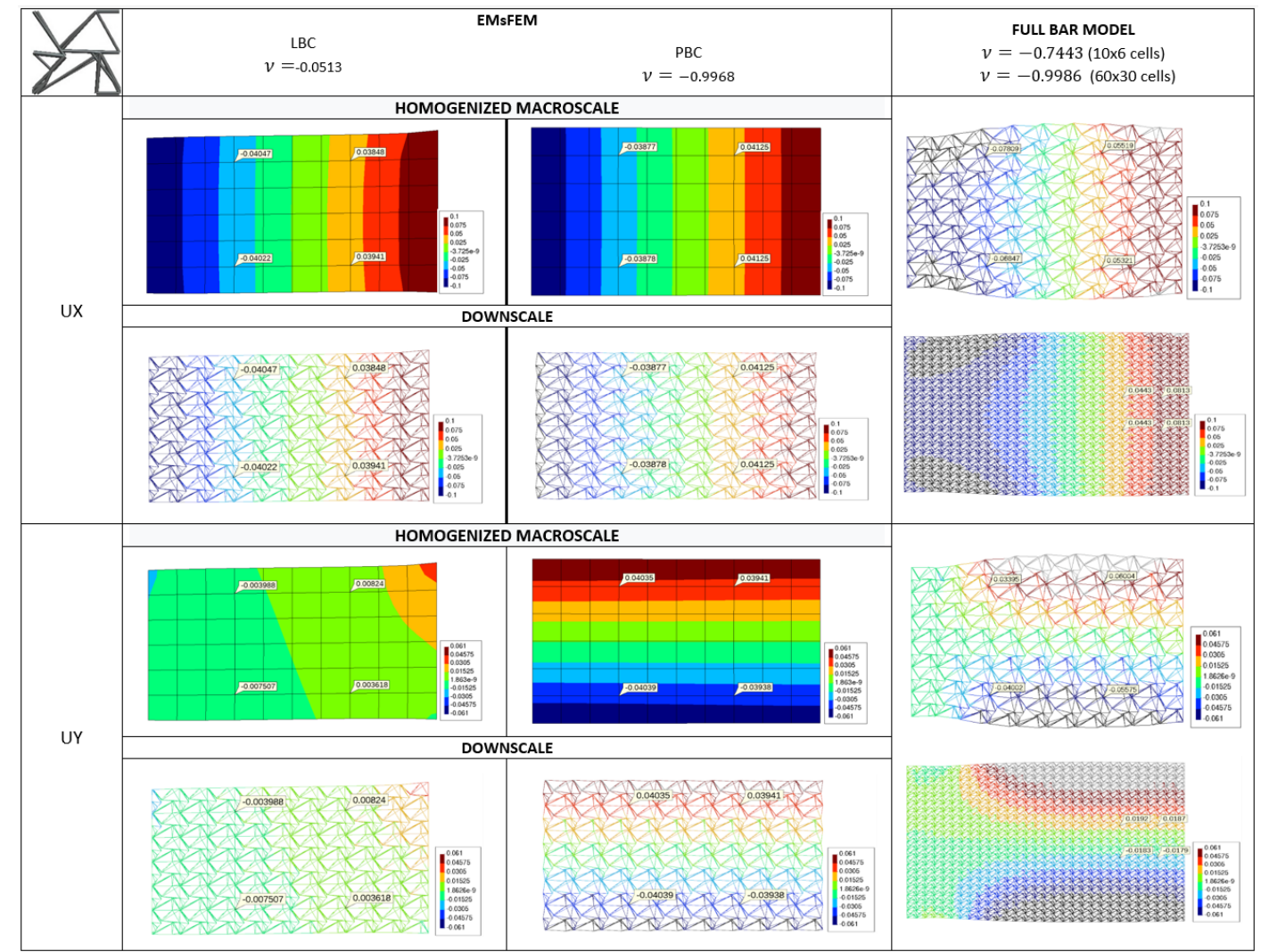

Figure 36 Min. Poisson ratio evaluated using EMsFEM - areas and coordinates plane stress FEM fully integrated linear quad. element $(A H)-v=-0.9977$.

\subsection{Evaluation of Poisson ratio of the PTMMs rotated $90^{\circ}$ clockwise}

According to Guth (2012) all the unit cells in this work would behave isotropically if inifinitesimal. Figure 37 displays a summary of the Poisson ratios obtained in the problem described in Section 6.5, but this time with each cell rotated 90 degrees clockwise. We remark that only approximate isotropy would be expected owing to size and border effects. Numerical experiments confirm approximately the same Poisson ratios. The Figure does not prove isotropy, it evaluates only two points in the respective polar plots. However, the coincidence of these results offers confidence in the $\mathrm{AH}$ isotropic prediction even for the finitely sized microscale of our problems.
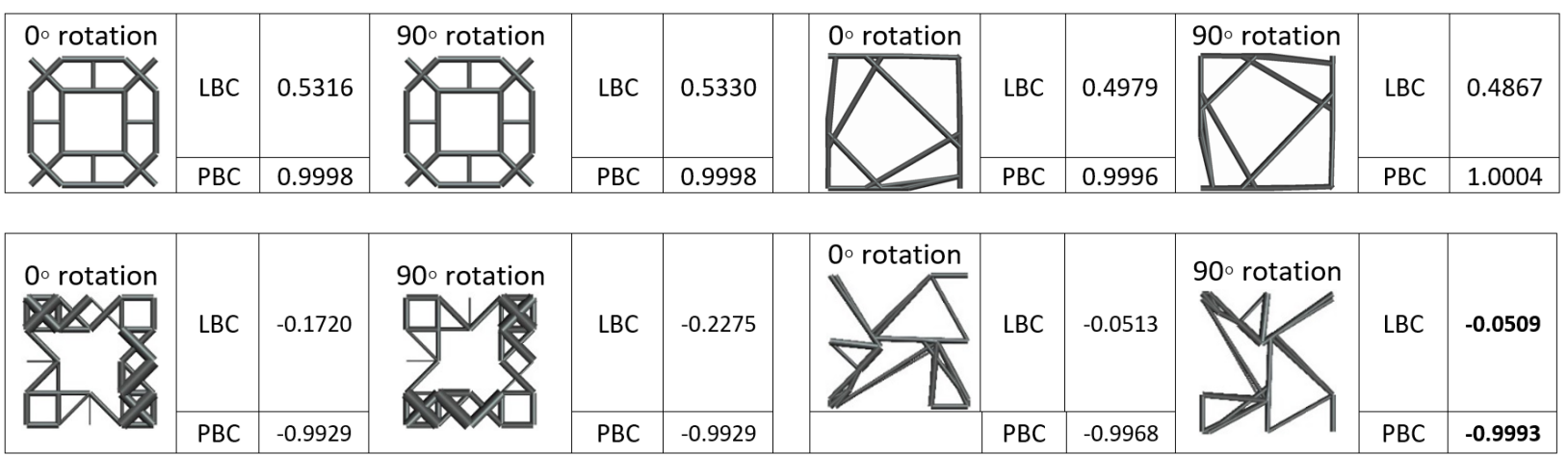

Figure 37 Evaluation of Poisson ratio for each cell rotated 90 clockwise for isotropy check. Although three cells are extremely non symmetric, the same Poisson value is confirmed in two directions.

\section{CONCLUDING REMARKS}

In this article we make a review of two tecnhiques commonly used to analyze the structural response of heterogeneous materials: asymptotic homogenization ( $\mathrm{AH}$ ) and the Extended Multicale Finite Element Method (EMsFEM). We use these methods to study periodic truss metamaterials (PTMMs) previously developed by Guth et al. 
(2012) to present extreme Poisson ratios and behave isotropically. The metamaterials were obtained under the premises of $\mathrm{AH}$, where the unit cell becomes infinitesimal and corresponds to a single material point. Numerical experiments were conducted in order to study the effect of a finitely sized microscale and the capability of the EMsFEM to approximate the macroscale physical behavior with a reduced model. To this end, two versions of the EMsFEM were implemented: the EMSFEM-LBC and the EMsFEM-PBC. The difference between them resides in the interpolation functions employed (linear and periodic boundary conditions). Some conclusions can be summarized:

(i) The macroscale problem defined for evaluation of the Poisson ratio consists of a coarse mesh, therefore size and border effects can be of great importance and this depends strongly on the unit cell and in macroscale boundary conditions;

(ii) In the case of the cells aimed at maximum Poisson ratio, the EMsFEM-PBC was able to reproduce adequately the full bar model. On the other hand, the EMsFEM-LBC led to a pronounced overstiffening effect, as expected;

(iii) In the case of the cells aimed at minimum Poisson ratio, nor the EMsFEM-LBC nor the EMsFEM-PBC were able to approximate the structural response of the full bar model. The former showed overstiffness and the later tended to the AH solution. Future analyses will study the oversampling technique (Zhang et al, 2010a);

(iv) Linear and periodic boundary conditions were applied to build numerical interpolation functions for each of the PTM unit cells in this work. Visual observation and the Frobenius norm of the homogenized EMsFEM stiffness matrices proved to be helpful to interpret the macroscopic behavior in each case.

(v) One of the unit cells, the one optimized for maximum Poisson ratio with areas and coordinates as design variables, showed spurious displacements in the interpolation functions. These spurious displacements are due to excessive compliance of the unit cell and could not be detected by AH. The reason is that the AH procedure is strain-driven and also assumes an infinitesimally sized unit cell. Spurious displacements can preclude the correct evaluation of failure criteria and nonlinear analyses using EMsFEM. On the other hand, they mean that the cell is excessively compliant and that large displacements are prone to occur in the domain of the full bar model. Hence, care must be taken before using PTMMs optimized via AH;

(vii) All the optimized unit cells studied in this work lead to isotropic macroscopic materials, provided the unit cell is infinitesimal. Three of them are completely asymmetric and isotropy is counterintuitive for finitely sized unit cells. We performed numerical tests to check the Poisson ratios for a 90 degree rotation of the unit cell, obtaining coincident results even though the micro/macro scale ratio is not small in the examples.;

\section{ACKNOWLEDGEMENTS}

The author Elias Jagiello acknowledges the support of the company Metal Crruzado for allowing partial leave of his regular duties in order to develop doctoral studies at Santa Catarina State University - UDESC.

Author's Contributions: all authors contributed equally.

Editor: Marco L. Bittencourt.

\section{References}

Arabnejad, S., Pasini, D. (2013). Mechanical properties of lattice materials via asymptotic homogenization and comparison with alternative homogenization methods. Int J Mech Sci 77:249-62.

Ashby, M.F. (1983).The mechanical properties of cellular solids. MTA 14: 1755-1769.

Cheng, L., Zhang, P., Biyikli, E., Bai, J., Robbins, J., To, A. (2017). Efficient design optimization of variable-density cellular structures for additive manufacturing: theory and experimental validation. Rapid Prototyp J 23(4):660-677

Cui, H., An, H., Huang, H. (2018). Truss topology optimization considering local buckling constraints and restrictions on intersection and overlap of bar members. Structural Multidisciplinary Optim. 58(2):575-594.

Gibson L. J., Ashby M. F. (1997). Cellular solids: Structure and properties. 2nd ed. United States: Cambridge solid state science series.

Glaesener, R., Lestringant, C., Telgen, B., Kochmann, D.M. (2019). Continuum models for stretching- and bending-dominated periodic trusses undergoing finite deformations, Int. J. Solids Struct 171:117-134. 
Guedes, J.M., Kikuchi, N. (1990). Preprocessing and postprocessing for materials based on the homogenization method with adaptive finite element methods. Computer Methods in Applied Mechanics and Engineering 83:143-198.

Guth, D.C. (2012). Optimization of truss-like cellular materials, including isotropy constraints, for thermomechanical applications, Master's Dissertation (in Portuguese), Universidade do Estado de Santa Catarina, Brazil.

Guth, D.C, Luersen, M.A., Muñoz-Rojas P.A. (2012). Optimization of periodic truss materials including constitutive symmetry constraints, Mat.-wiss. u.Werkstofftech 43(5): 447-456.

Guth, D.C, Luersen, M.A, Muñoz-Rojas, P.A. (2015). Optimization of three-dimensional truss-like periodic materials considering isotropy constraints, Struct Multidisc Optim 52:889-901.

Hassani, B., Hinton, E. (1998). A review of homogenization and topology optimization I - Homogenization theory for media with periodic structure. Computers and Structures 69:707-717.

Hou, T.Y., Wu, X.H. (1996). A multiscale finite element method for elliptic problems in composite materials and porous media. Journal of computational physics 134(1):169-189.

Jun, Y., Wenbo, H., Zunyi, D. (2015). Structure/material concurrent optimization of lattice materials based on extended multiscale finite element method, Journal for Multiscale Computational Engineering 13(1): 73-90.

Li, D.W., Liao, W.H., Dai, N., Xie, Y.M. (2020). Anisotropic design and optimization of conformal gradient lattice structures, Computer- Aided Design, 119 - 102787.

Liu, H., Lv, J. (2017). An equivalent continuum multiscale formulation for 2D geometrical nonlinear analysis of lattice truss structure, Compos. Struct 160:335-348.

Liu, L., Yan, J., Cheng, G. (2008). Optimum structure with homogeneous optimum truss-like material, Computers and Structures 86:1417-1425.

Muñoz-Rojas, P.A., Carniel, T.A., Silva, E.C.N., Öchsner, A. (2010). Optimization of a unit periodic cell in lattice block materials aimed at thermo-mechanical applications, in A. Öchsner and G.E. Murch (eds.), Heat Transfer in Multi-Phase Materials, Adv Struct Mater 2, Springer-Verlag Berlin Heidelberg.

Neves, M.M., Rodrigues, H., Guedes, J.M. (2000). Comput. Struct 76(1-3): 421-429.

Otero, F., Oller, S., Martinez, X. (2018). Multiscale computational homogenization: review and proposal of a new enhancedfirst-order method, Arch. Comput. Methods Eng 25(2):479-505.

Peric,'D., de Souza Neto, E.A., Feijoó, R.A., Partovi, M., Molina, A.J.C. (2011). On micro-to-macro transitions for multi-scale analysis ofnon-linear heterogeneous materials: unified variational basis and finite element implementation. Int J Numer Methods Eng 87(1-5):149-170.

Plocher, J., Panesar, A. (2019). Review on design and structural optimisation in additive manufacturing: towards nextgeneration lightweight structures. Mater Des 183 - 108164.

Schittkowski, K. (2006). NLPQLP: A Fortran implementation of a sequential quadratic programming algorithm with distributed and non-monotone line Search - User's guide, Version 2.2, Report, Department of Computer Science, University of Bayreuth.

Sigmund, O. (1994), Materials with prescribed constitutive parameters: an inverse homogenization problem, Int. J. Solids Structures 31(17):2313-2329.

Sigmund, O. (1995). Tailoring materials with prescribed elastic properties, Mechanics of Materials $20351-368$

Sun, C.T., Vaidya, R.S. (1996). Prediction of composite properties from a representative volume element. Composite Science and Technology 56:171-179.

Xia, Z.H., Zhou, C.W., Yong, Q.L., Wang, X.W. (2006). On selection of repeated unit cell model and application of unified periodic boundary conditions in micromechanical analysis of composites. Int J Solid Struct 43(2):266-78.

Yan, J., Cheng, G., Liu, S., Liu, L. (2006). Comparison of prediction on effective elastic property and shape optimization of truss material with periodic microstructure. International Journal of Mechanical Sciences 10:400-413.

Yan, J., Yang, S., Duan, Z., Yang, C. (2015). Minimum compliance optimization of a thermoelastic lattice structure with sizecoupled effects. Journal of Thermal Stresses, 38(3):338-357. 
Zhang, H.W, Fu, Z.D., Wu, J.K. (2009). Coupling multiscale finite element method for consolidation analysis of heterogeneous saturated porous media. Advances in Water Resources 32(2):268-279.

Zhang, W., Sun, S. (2006). Scale-related topology optimization of cellular materials and structures, Int. J. Numer. Meth. Engng; 68:993-1011.

Zhang, H.W., Wu, J.K., Fu, Z.D. (2010a). Extended multiscale finite element method for mechanical analysis of periodic lattice truss materials. Journal for Multiscale Computational Engineering 8:597-613.

Zhang, H.W, Wu, J.K, Lü, J., Fu, Z.D. (2010b). Extended multiscale finite element method for mechanical analysis of heterogeneous materials, Acta Mech Sin 26:899-920.

Wang, C., Gu, X., Zhu, J., Zhou, H., Li, S., Zhang, W. (2020). Concurrent design of hierarchical structures with three-dimensional parameterized lattice microstructures for additive manufacturing. Structural and Multidisciplinary Optimization 61:869-894. 


\section{APPENDIX 1}

In this Appendix we list the stiffness matrices obtained in Section 5 for the EMsFEM with loinear and periodic boundary conditions. We also present their Frobenius norms with the percentual differences.

7.1. Initial cell

$$
\begin{aligned}
& \boldsymbol{K}_{8 \times 8}^{L B C}=\left[\begin{array}{rrrrrrrr}
54.68 & 15.09 & -38.38 & -1.21 & -19.67 & -15.09 & 3.36 & 1.21 \\
15.09 & 54.68 & 1.21 & 3.36 & -15.09 & -19.67 & -1.21 & -38.38 \\
-38.38 & 1.21 & 54.68 & -15.09 & 3.36 & -1.21 & -19.67 & 15.09 \\
-1.21 & 3.36 & -15.09 & 54.68 & 1.21 & -38.38 & 15.09 & -19.67 \\
-19.67 & -15.09 & 3.36 & 1.21 & 54.68 & 15.09 & -38.38 & -1.21 \\
-15.09 & -19.67 & -1.21 & -38.38 & 15.09 & 54.68 & 1.21 & 3.36 \\
3.36 & -1.21 & -19.67 & 15.09 & -38.38 & 1.21 & 54.68 & -15.09 \\
1.21 & -38.38 & 15.09 & -19.67 & -1.21 & 3.36 & -15.09 & 54.68
\end{array}\right] \times 10^{6} \mathrm{~N} / \mathrm{mm} \\
& \boldsymbol{K}_{8 \times 8}^{P B C}=\left[\begin{array}{rrrrrrrr}
50.63 & 12.77 & -38.61 & 0.75 & -18.89 & -12.77 & 6.86 & -0.75 \\
12.77 & 50.63 & -0.75 & 6.86 & -12.77 & -18.89 & 0.75 & -38.61 \\
-38.61 & -0.75 & 50.63 & -12.77 & 6.86 & 0.75 & -18.89 & 12.77 \\
0.75 & 6.86 & -12.77 & 50.63 & -0.75 & -38.61 & 12.77 & -18.89 \\
-18.89 & -12.77 & 6.86 & -0.75 & 50.63 & 12.77 & -38.61 & 0.75 \\
-12.77 & -18.89 & 0.75 & -38.61 & 12.77 & 50.63 & -0.75 & 6.86 \\
6.86 & 0.75 & -18.89 & 12.77 & -38.61 & -0.75 & 50.63 & -12.77 \\
-0.75 & -38.61 & 12.77 & -18.89 & 0.75 & 6.86 & -12.77 & 50.63
\end{array}\right] \times 10^{6} \mathrm{~N} / \mathrm{mm}
\end{aligned}
$$

$\left\|K_{L B C}\right\|=0.20631202 \times 10^{9} \mathrm{~N} / \mathrm{mm} ;$

$\left\|K_{P B C}\right\|=0.19567868 \times 10^{9} \mathrm{~N} / \mathrm{mm}$;

$E_{K}=5.4 \%$.

7.2. Maximum Poisson ratio - areas

$$
\begin{aligned}
& \boldsymbol{K}_{8 \times 8}^{L B C}=\left[\begin{array}{rrrrrrrr}
40.17 & 17.56 & -30.85 & 8.24 & -17.56 & -17.56 & 8.24 & -8.24 \\
17.56 & 40.3 & -8.24 & 8.23 & -17.56 & -17.56 & 8.24 & -30.98 \\
-30.85 & -8.24 & 40.17 & -17.56 & 8.23 & 8.24 & -17.56 & 17.56 \\
8.24 & 8.23 & -17.56 & 40.3 & -8.24 & -30.98 & 17.56 & -17.56 \\
-17.56 & -17.56 & 8.23 & -8.24 & 40.17 & 17.56 & -30.85 & 8.24 \\
-17.56 & -17.56 & 8.24 & -30.98 & 17.56 & 40.3 & -8.24 & 8.23 \\
8.24 & 8.24 & -17.56 & 17.56 & -30.85 & -8.24 & 40.17 & -17.56 \\
-8.24 & -30.98 & 17.56 & -17.56 & 8.24 & 8.23 & -17.56 & 40.3
\end{array}\right] \times 10^{6} \mathrm{~N} / \mathrm{mm} \\
& \boldsymbol{K}_{8 \times 8}^{P B C}=\left[\begin{array}{rrrrrrrr}
29.77 & 18.47 & -29.77 & 18.46 & -7.17 & -18.47 & 7.17 & -18.46 \\
18.47 & 29.84 & -18.46 & 7.1 & -18.47 & -7.11 & 18.46 & -29.83 \\
-29.77 & -18.46 & 29.77 & -18.47 & 7.17 & 18.46 & -7.17 & 18.47 \\
18.46 & 7.1 & -18.47 & 29.84 & -18.46 & -29.83 & 18.47 & -7.11 \\
-7.17 & -18.47 & 7.17 & -18.46 & 29.77 & 18.47 & -29.77 & 18.46 \\
-18.47 & -7.11 & 18.46 & -29.83 & 18.47 & 29.84 & -18.46 & 7.1 \\
7.17 & 18.46 & -7.17 & 18.47 & -29.77 & -18.46 & 29.77 & -18.47 \\
-18.46 & -29.83 & 18.47 & -7.11 & 18.46 & 7.1 & -18.47 & 29.84
\end{array}\right] \times 10^{6} \mathrm{~N} / \mathrm{mm}
\end{aligned}
$$

$\left\|K_{L B C}\right\|=0.17214765 \times 10^{9} \mathrm{~N} / \mathrm{mm} ;$

$\left\|K_{P B C}\right\|=0.16109491 \times 10^{9} \mathrm{~N} / \mathrm{mm}$;

$E_{K}=6.8 \%$. 
7.3. Maximum Poisson ratio - areas and coordinates

$$
\begin{aligned}
& \boldsymbol{K}_{8 \times 8}^{L B C}=\left[\begin{array}{rrrrrrrr}
48.25 & 29.84 & -35.09 & -3.61 & -11.09 & -9.54 & -2.06 & -16.68 \\
29.84 & 50.66 & -17.06 & -1.91 & -9.55 & -10.86 & -3.22 & -37.88 \\
-35.09 & -17.06 & 44.79 & -11.79 & 4.21 & 2.09 & -13.91 & 26.76 \\
-3.61 & -1.91 & -11.79 & 63.89 & -16.87 & -48.48 & 32.28 & -13.49 \\
-11.09 & -9.55 & 4.21 & -16.87 & 60.11 & 23.75 & -53.24 & 2.67 \\
-9.54 & -10.86 & 2.09 & -48.48 & 23.75 & 55.93 & -16.3 & 3.41 \\
-2.06 & -3.22 & -13.91 & 32.28 & -53.24 & -16.3 & 69.22 & -12.76 \\
-16.68 & -37.88 & 26.76 & -13.49 & 2.67 & 3.41 & -12.76 & 47.97
\end{array}\right] \times 10^{6} \mathrm{~N} / \mathrm{mm} \\
& \boldsymbol{K}_{8 \times 8}^{P B C}=\left[\begin{array}{rrrrrrrr}
21.81 & 14 & -21.8 & 4.9 & 2.94 & -4.91 & -2.95 & -13.99 \\
14 & 23.5 & -14 & -3.21 & -6.24 & 3.21 & 6.24 & -23.5 \\
-21.8 & -14 & 21.79 & -4.9 & -2.94 & 4.9 & 2.94 & 14 \\
4.9 & -3.21 & -4.9 & 36.63 & -28.54 & -36.63 & 28.54 & 3.21 \\
2.94 & -6.24 & -2.94 & -28.54 & 37.79 & 28.54 & -37.79 & 6.25 \\
-4.91 & 3.21 & 4.9 & -36.63 & 28.54 & 36.64 & -28.53 & -3.22 \\
-2.95 & 6.24 & 2.94 & 28.54 & -37.79 & -28.53 & 37.8 & -6.25 \\
-13.99 & -23.5 & 14 & 3.21 & 6.25 & -3.22 & -6.25 & 23.51
\end{array}\right] \times 10^{6} \mathrm{~N} / \mathrm{mm}
\end{aligned}
$$

$\left\|K_{L B C}\right\|=0.22703051 \times 10^{9} \mathrm{~N} / \mathrm{mm}$;

$\left\|K_{P B C}\right\|=0.15472082 \times 10^{9} \mathrm{~N} / \mathrm{mm}$;

$E_{K}=46.7 \%$.

7.4. Minimum Poisson ratio - areas

$$
\begin{aligned}
& \boldsymbol{K}_{8 \times 8}^{L B C}=\left[\begin{array}{rrrrrrrr}
57.52 & 27 & -23.04 & 0.05 & -35.02 & -34.53 & 0.53 & 7.48 \\
27 & 60.99 & 6.79 & 0.89 & -33.87 & -34.69 & 0.07 & -27.19 \\
-23.04 & 6.79 & 34.84 & -10.08 & -6.19 & -1.71 & -5.6 & 5 \\
0.05 & 0.89 & -10.08 & 49.38 & 4.87 & -39.35 & 5.15 & -10.92 \\
-35.02 & -33.87 & -6.19 & 4.87 & 71.76 & 36.34 & -30.54 & -7.34 \\
-34.53 & -34.69 & -1.71 & -39.35 & 36.34 & 75.61 & -0.08 & -1.56 \\
0.53 & 0.07 & -5.6 & 5.15 & -30.54 & -0.08 & 35.61 & -5.14 \\
7.48 & -27.19 & 5 & -10.92 & -7.34 & -1.56 & -5.14 & 39.68
\end{array}\right] \times 10^{6} \mathrm{~N} / \mathrm{mm} \\
& \boldsymbol{K}_{8 \times 8}^{P B C}=\left[\begin{array}{rrrrrrrr}
14.72 & -3.74 & -12.7 & -1.4 & 7.52 & -0.61 & -9.54 & 5.76 \\
-3.74 & 17.3 & 5.9 & -12.09 & -0.71 & 9.92 & -1.44 & -15.13 \\
-12.7 & 5.9 & 18.64 & -0.76 & -13.47 & -5.18 & 7.53 & 0.04 \\
-1.4 & -12.09 & -0.76 & 14.87 & 3.51 & -12.7 & -1.34 & 9.92 \\
7.52 & -0.71 & -13.47 & 3.51 & 16.3 & 2.43 & -10.35 & -5.23 \\
-0.61 & 9.92 & -5.18 & -12.7 & 2.43 & 18.5 & 3.36 & -15.72 \\
-9.54 & -1.44 & 7.53 & -1.34 & -10.35 & 3.36 & 12.37 & -0.57 \\
5.76 & -15.13 & 0.04 & 9.92 & -5.23 & -15.72 & -0.57 & 20.94
\end{array}\right] \times 10^{6} \mathrm{~N} / \mathrm{mm}
\end{aligned}
$$

$\left\|K_{L B C}\right\|=0.21598578 \times 10^{9} \mathrm{~N} / \mathrm{mm}$;

$\left\|K_{P B C}\right\|=0.76840042 \times 10^{8} \mathrm{~N} / \mathrm{mm}$;

$E_{K}=181 \%$. 
7.5. Minimum Poisson ratio - areas and coordinates

$$
\begin{aligned}
& \boldsymbol{K}_{8 \times 8}^{L B C}=\left[\begin{array}{rrrrrrrr}
20.16 & 5.98 & -12.74 & -7.17 & -6.94 & -0.23 & -0.46 & 1.43 \\
5.98 & 34.97 & 2.12 & -5.71 & -5.27 & -2.39 & -2.83 & -26.86 \\
-12.74 & 2.12 & 21.24 & -2.8 & 0.59 & -5.69 & -9.08 & 6.37 \\
-7.17 & -5.71 & -2.8 & 13.07 & 7.58 & -3.09 & 2.39 & -4.26 \\
-6.94 & -5.27 & 0.59 & 7.58 & 14.86 & -1.22 & -8.5 & -1.08 \\
-0.23 & -2.39 & -5.69 & -3.09 & -1.22 & 9.02 & 7.15 & -3.53 \\
-0.46 & -2.83 & -9.08 & 2.39 & -8.5 & 7.15 & 18.05 & -6.71 \\
1.43 & -26.86 & 6.37 & -4.26 & -1.08 & -3.53 & -6.71 & 34.67
\end{array}\right] \times 10^{6} \mathrm{~N} / \mathrm{mm} \\
& \boldsymbol{K}_{8 \times 8}^{P B C}=\left[\begin{array}{rrrrrrrr}
12.54 & -1.74 & -5.93 & -5.94 & -1.74 & -0.66 & -4.85 & 8.34 \\
-1.74 & 8.68 & 7.73 & -0.81 & 0.12 & -5.17 & -6.12 & -2.68 \\
-5.93 & 7.73 & 11.28 & -0.05 & -3.59 & -5.28 & -1.74 & -2.39 \\
-5.94 & -0.81 & -0.05 & 4.9 & 4.13 & 1.09 & 1.87 & -5.18 \\
-1.74 & 0.12 & -3.59 & 4.13 & 7.85 & 1.21 & -2.51 & -5.47 \\
-0.66 & -5.17 & -5.28 & 1.09 & 1.21 & 4.85 & 4.73 & -0.76 \\
-4.85 & -6.12 & -1.74 & 1.87 & -2.51 & 4.73 & 9.11 & -0.48 \\
8.34 & -2.68 & -2.39 & -5.18 & -5.47 & -0.76 & -0.48 & 8.64
\end{array}\right] \times 10^{6} \mathrm{~N} / \mathrm{mm}
\end{aligned}
$$

$\left\|K_{L B C}\right\|=0.84625235 \times 10^{8} \mathrm{~N} / \mathrm{mm} ;$

$\left\|K_{P B C}\right\|=0.39372490 \times 10^{8} \mathrm{~N} / \mathrm{mm} ;$

$E_{K}=115 \%$. 